\title{
Planning and Provisioning Strategies for Optical Core Networks
}

\author{
Ajmal Muhammad
}

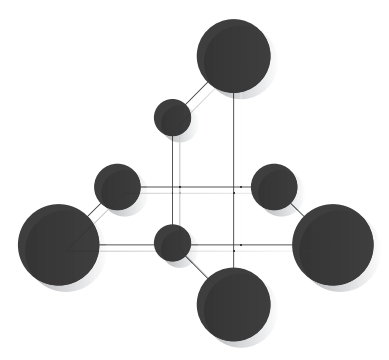

INFORMATION CODING Linköping University

Division of Information Coding

Department of Electrical Engineering

Linköping University

SE-581 83 Linköping, Sweden

www.icg.isy.liu.se

Linköping 2015 
Linköping Studies in Science and Technology

Dissertations No. 1645

Ajmal Muhammad muhammad.ajmal@liu.se

www.icg.isy.liu.se

Division of Information Coding

Department of Electrical Engineering

Linköping University

SE-581 83 Linköping, Sweden

Planning and Provisioning Strategies for Optical Core Networks

(c) 2015 Ajmal Muhammad, unless otherwise noted.

ISBN 978-91-7519-115-7

ISSN 0345-7524

Printed in Sweden by LiU-Tryck, Linköping 2015 


\section{Abstract}

Optical communication networks are considered the main catalyst for the transformation of communication technology, and serve as the backbone of today's Internet. The inclusion of exciting technologies, such as, optical amplifiers, wavelength division multiplexing (WDM), and reconfigurable optical add/drop multiplexers (ROADM) in optical networks have made the cost of information transmission around the world negligible. However, to maintain the cost effectiveness for the growing bandwidth demand, facilitate faster provisioning, and provide richer sets of service functionality, optical networks must continue to evolve. With the proliferation of cloud computing the demand for a promptly responsive network has increased. Moreover, there are several applications, such as, real time multimedia services that can become realizable, depending on the achievable connection set-up time.

Given the high bandwidth requirements and strict service level specifications (SLSs) of such applications, dynamic on-demand WDM networks are advocated as a first step in this evolution. SLSs are metrics of a service level agreement (SLA), which is a contract between a customer and network operator. Apart from the other candidate parameters, the set-up delay tolerance, and connection holding-time have been defined as metrics of SLA. Exploiting these SLA parameters for on-line provisioning strategies exhibits a good potential in improving the overall network blocking performance. However, in a scenario where connection requests are grouped in different service classes, the provisioning success rate might be unbalanced towards those connection requests with less stringent requirements, i.e., not all the connection requests are treated in a fair way.

The first part of this thesis focuses on different scheduling strategies for promoting the requests belonging to smaller set-up delay tolerance service classes. The first part also addresses the problem of how to guarantee the signal quality and the fair provisioning of different service classes, where 
each class corresponds to a specified target of quality of transmission. Furthermore, for delay impatient applications the thesis proposes a provisioning approach, which employs the possibility to tolerate a slight degradation in quality of transmission during a small fraction of the holding-time.

The next essential phase for scaling system capacity and satisfying the diverse customer demands is the introduction of flexibility in the underlying technology. In this context, the new optical transport networks, namely elastic optical networks (EON) are considered as a worthwhile solution to efficiently utilize the available spectrum resources. Similarly, space division multiplexing (SDM) is envisaged as a promising technology for the capacity expansion of future networks. Among the alternative for flexible nodes, the architecture on demand ( $\mathrm{AoD}$ ) node has the capability to dynamically adapt its composition according to the switching and processing needs of the network traffic.

The second part of this thesis investigates the benefits of set-up delay tolerance for EON by proposing an optimization model for dynamic and concurrent connection provisioning. Furthermore, it also examines the planning aspect for flexible networks by presenting strategies that employ the adaptability inherent in AoD. Significant reduction in switching devices is attainable by proper planning schemes that synthesized the network by allocating switching device where and when needed while maximizing fiber switching operation. In addition, such a design approach also reduces the power consumption of the network. However, cost-efficient techniques in dynamic networks can deteriorate the network blocking probability owing to insufficient number of switching modules. For dynamic networks, the thesis proposes an effective synthesis provisioning scheme along with a technique for optimal placement of switching devices in the network nodes.

The network planning problem is further extended to multi-core-fiber (MCF) based SDM networks. The proposed strategies for SDM networks aim to establish the connections through proper allocation of spectrum and core while efficiently utilizing the spectrum resources. Finally, the optimal planning strategy for SDM networks is tailored to fit synthetic AoD based networks with the goal to optimally build each node and synthesize the whole network with minimum possible switching resources. 


\section{Sammanfattning}

Optiska kommunikationsnät ses som den viktigaste katalysatorn för den förändring som sker inom kommunikationstekniken, främst vad avser Internets kärnnät. Införandet av teknologier såsom optiska förstärkare, våglängdsmultiplexering (WDM) och konfigurerbara optiska multiplexerare (ROADM) i optiska nätverk har minskat kostnaden för världsomspännande informationsöverföring till negligerbara nivåer. För att kunna bibehålla denna kostnadseffektivitet när behovet av bandbredd ökar så behöver fortsatt utveckling ske mot snabbare förbindelser och fler funktioner. Med den ökade spridningen av "molntjänster" ökar också behovet av ett snabbt nätverk med låg fördröjning. Till detta kan läggas att det finns ett antal tillämpningar såsom realtids multimediatjänster som kan bli alltmer aktuella beroende på vilka faktiska uppkopplingstider som kan åstadkommas.

Dynamiska WDM-nätverk har rekommenderats som ett första steg i utvecklingen mot tjänster som kräver hög bandbredd och har strikta krav (sk SLS) på servicenivån. Med SLS menas de specifikationer som ligger till grund för avtalet (SLA) mellan nätverksoperatör och användare. Två specifika parametrar i SLA är den av användaren accepterade tiden för uppkoppling samt uppskattningen av hur länge varje uppkoppling ska pågå. Genom att utnyttja dessa parametrar vid fördelningen av nätverksresurserna i ett dynamiskt scenario så kan man uppnå stora förbättringar och minska risken för att uppkopplingar blockeras på grund av brist på resurser. Det har dock visat sig att i ett scenario där uppkopplingar tillhör olika serviceklasser så riskerar tillgängligheten att bli obalanserad. Uppkopplingar med lägre servicekrav kommer att favoriseras, dvs samtliga uppkopplingar kommer inte att behandlas på ett rättvist sätt.

Första delen av avhandlingen fokuserar på uppkopplingsstrategier för att gynna användare som har striktare servicekrav rörande uppkopplingstid. Denna del adresserar också problemet hur man kan garantera signalkvaliteten 
och åstadkomma rättvis tilldelning av nätverksresurser till olika serviceklasser där varje serviceklass motsvarar en viss transmissionskvalitet. Vidare behandlas fallet där användaren kan acceptera en liten försämring i transmissionskvalitet under en kort tid för att därmed uppnå snabbare uppkopplingstid.

Nästa stora förändring för att kunna skala upp kapaciteten och tillfredsställa de olika framtida användarkraven är införandet av flexibilitet i den underliggande nätverksteknologin. Nya "elastiska" optiska transportnät (EON) ses som en möjlig väg framåt för att ännu effektivare kunna utnyttja de tillgängliga spektrumresurserna. På motsvarande sätt ses rumsmässig multiplexering (SDM) som en lovande teknologi för att komma förbi kapacitetsbegränsningarna i dagens optiska nätverk. Bland de alternativ som finns för att implementera flexibla nätverksnoder har "Architecture on Demand" (AoD) visat sig ha förmågan att kunna uppfylla de krav som ställs på sådana framtida noder.

Den andra delen av avhandlingen studerar de fördelar som kan uppnås när användarens tolerans för längre uppkopplingstider kombineras med EON via en föreslagen optimeringsmetod för dynamiska uppkopplingar. I denna del diskuteras även planeringsaspekten för flexibla nätverk då strategier används som utnyttjar adaptiviteten i AoD. En signifikant minskning i antalet omkopplingsenheter kan uppnås i ett "syntetiserat" nätverk genom att allokera enheter bara då de behövs. Som ytterligare bonus uppnås en lägre energiförbrukning. Dock kan sådana kostnadseffektiva metoder leda till en ökad risk för att uppkopplingar blockeras beroende på det mindre antalet tillgängliga omkopplingsenheter. Avhandlingen föreslår en effektiv syntesmetod för att tilldela resurser kombinerat med målet att uppnå optimal placering av omkopplingsenheter i nätverksnoderna.

Planeringsproblemet är vidare utökat till att omfatta SDM-nätverk baserade på fibrer som innehåller multipla kärnor $(\mathrm{MCF})$. De föreslagna strategierna för SDM-nätverk syftar till att etablera förbindelser genom lämplig allokering av såväl spektrum som fiberkärnor med effektiv användning av spektrumresurserna. Den optimala planeringsstrategin för SDM-nätverk anpassas slutligen till syntetiska AoD-baserade nätverk med målet att finna optimala nodkonfigurationer och därmed syntetisera hela nätverket med minsta möjliga antal switchresurser. 


\section{Acknowledgments}

It is a pleasure to look back and recall all those wonderful people who have supported me in this long but fascinating doctoral journey. Without their assistance and encouragement, this dissertation would have remained a distant dream for me.

First and foremost, I would like to express my special appreciation and thanks to my main supervisor Prof. Robert Forchheimer for his valuable guidance, patience, care, and providing an excellent environment for doing research. Robert is a person with an amicable and positive disposition, and I consider it as an honor to be his student.

My sincere gratitude is also reserved for my co-supervisor Prof. Lena Wosinska at KTH Royal Institute of technology. Lena always encouraged me to benefit from the expertise of her prestigious research group. Besides, I am really thankful for her aspiring guidance, invaluably constructive criticism and friendly advice during these years.

I express my warm thanks to Dr. Paolo Monti and Dr. Cicek Cavdar at School of ICT, KTH for their support, guidance, and critical discussions through the years of my Ph.D. study. I am also indebted to Dr. Isabella Cerutti at Scuola Superiore Sant' Anna, Pisa for her precious collaboration and technical support.

I would like to extend my gratitude to Prof. Dimitra Simeonidou, Dr. Georgios Zervas, and Dr. Noberto Amaya at University of Bristol for generously allowing me to visit their distinguished research group. This visit led to a fruitful collaboration, which significantly enriched the scope of the present thesis. Special thanks to all the co-authors of my publications for their valuable cooperation and technical assistance.

I am grateful to my colleagues and professors at Information Coding Division for creating an enjoyable working atmosphere, and for interesting discussions 
during fikas. Thanks to Carina Lindström for taking care of the administrative issues.

I would like to thank Vinnova (The Swedish Governmental Agency for Innovation Systems) for the financial support under the projects of "All-Optical Overlay Networks" and "Security in All-Optical Networks".

Heartfelt thanks to all my friends, especially those who made this experience memorable, in particular, Dr. Iqbal Hussain, Rabiullah Khattak, Dr. Abdul Naeem, and Dr. Aftab Ahmad. I would also like to thanks my friend Putri Sarah Sembiring for her care and love.

Finally, but most importantly, I would like to direct my warmest thanks to my loving and caring family. I am greatly indebted to my family for their everlasting love, understanding, and patience.

Ajmal Muhammad Linköping, March 2015 


\section{List of Acronyms}

ADC Analog to Digital Converter

AoD Architecture on Demand

AR Advance Reservation

ASE Amplified Spontaneous Emission

ASON Automatically Switched Optical Network

AWG Arrayed Waveguide Grating

BBR Bandwidth Blocking Ratio

BER Bit Error Rate

BP Blocking Probability

BS Broadcast-and-Select

BVT Bandwidth Variable Transceiver

CD Chromatic Dispersion

CDC Colorless, Directionless, and Contentionless

CR-LDP Constraint based Routing with Label Distribution Protocol

DAC Digital to Analog Converter

DP-PSSS Dynamic Provisioning with Preemptable Spectrum Selective Switches

EBP Elastic Bulk Provisioning

EDFA Erbium Doped Fiber Amplifier

EON Elastic Optical Network

FEC Forward Error Correction

FTTP Fiber To The Premises 
FWM Four Wave Mixing

GMPLS Generalized Multi-Protocol Label Switching

GoS Grade of Service

IETF Internet Engineering Task Force

ILP Integer Linear Programming

IR Immediate Reservation

ITU International Telecommunication Union

LCoS Liquid Crystal on Silicon

LMP Link Management Protocol

MCF Multi Core Fiber

MG-OXC Multi Granular Optical Cross Connect

MMF Multi Mode Fiber

MEMS Micro-Electro-Mechanical System

NDT No Delay Tolerance

NNI Network-to-Network Interface

N-WDM Nyquist Wavelength Division Multiplexing

OADM Optical Add/Drop Multiplexer

OBS Optical Burst Switching

OCS Optical Circuit Switching

OEO Optical-Electronic-Optical

O-OFDM Optical Orthogonal Frequency Division Multiplexing

OPS Optical Packet Switching

OSNR Optical Signal-to-Noise Ratio

OSPF-TE Open Shortest Path First with Traffic Engineering

O-VPN Optical Virtual Private Network

OXC Optical Cross Connect

PCE Path Computation Element

PDL Polarization Dependent Losses

PDT Provision with Delay Tolerance

PLI Physical Layer Impairments 
PMD Polarization Mode Dispersion

QoP Quality of Protection

QoS Quality of Service

QoT Quality of Transmission

ROADM Reconfigurable Optical Add/Drop Multiplexer

RSA Routing and Spectrum Allocation

RSVT-TE Resource Reservation Protocol with Traffic Engineering

RWA Routing and Wavelength Assignment

SBS Stimulated Raman Scattering

SBVT Sliceable Bandwidth Variable Transceiver

SC Service Class

SDM Space Division Multiplexing

SDN Software-Defined Networking

SLA Service Level Agreement

SLS Service Level Specification

SMF Single Mode Fiber

SNR Signal-to-Noise Ratio

SOA Semiconductor Optical Amplifier

SPFF Shortest Path with First Fit

SPFS Shortest Path with Minimum Fiber Switch

SPM Self Phase Modulation

SR Spectrum Routing

SSS Spectrum-Selective Switch

TDM Time Division Multiplexing

UHDTV Ultra-high Definition TV

UNI User-to-Network Interface

VoD Video on Demand

WDM Wavelength Division Multiplexing

WSS Wavelength-Selective Switch

XPM Cross Phase Modulation 


\section{Contents}

Abstract

Sammanfattning v v

Acknowledgements vii

List of Acronyms ix

$\begin{array}{ll}\text { Contents } & \text { xiii }\end{array}$

I Introduction 1

1 Introduction $\quad 3$

1.1 Optical Core Networks . . . . . . . . . . . . . . . 3

1.1.1 Dynamic Optical Networks . . . . . . . . . . . . 4

1.1.2 Flexible Optical Networks . . . . . . . . . . . . . 5

1.1.3 Space Division Multiplexing . . . . . . . . . . 6

1.2 Optical Switching . . . . . . . . . . . . . 7

1.2.1 Optical Circuit Switching . . . . . . . . . . 7

1.2.2 Optical Packet Switching . . . . . . . . . . . 8

1.2.3 Optical Burst Switching . . . . . . . . . . . . . 9

1.3 Methodology for Performance Evaluation of Optical Networks 10

1.3.1 Discrete Event Driven Simulation . . . . . . . . . . 10

1.4 Organization of the Thesis . . . . . . . . . . . . 10

2 Provisioning in Wavelength Routed Optical Networks 13

2.1 The RWA Problem . . . . . . . . . . . . . . . . 15

2.1.1 Physical Layer Impairments . . . . . . . . . . . . . 15

2.1.2 Traffic Models. . . . . . . . . . . . . . . . . . . 16

2.1.3 Approaches for Solving the RWA Problem . . . . . . . 17

2.1.4 RWA Algorithms for Ideal Physical Layer . . . . . . . 18

2.1.5 RWA Algorithms for Non-Ideal Physical Layer . . . . 19

2.2 Optical Network Control and Management . . . . . . . . . . . 21

2.2.1 Control Architecture for RWA . . . . . . . . . . . . . 21

2.2.2 Standards for Control Plane . . . . . . . . . . . . . 22 
2.3 Differentiated Classes of Service in Optical Networks . . . . . 23

2.4 Time Dimension Issues for Connections Provisioning . . . . . . 24

2.4.1 Scheduled or Advance Reservation . . . . . . . . . . 25

2.4 .2 Holding-time . . . . . . . . . . . . . . . 25

2.4.3 Set-up Delay Tolerance . . . . . . . . . . . . . 26

2.5 Quality of Transmission . . . . . . . . . . . 28

3 Elastic Optical Networking 31

3.1 Elastic Optical Path . . . . . . . . . . . . . . . . . . 32

3.1 .1 The Offline RSA Problem . . . . . . . . . . . . 33

3.1 .2 The Online RSA Problem . . . . . . . . . . . . . 34

3.2 Node Architecture for Elastic Optical Transport . . . . . . . 35

3.3 Flexible Transceivers . . . . . . . . . . . . . . . . . 38

3.4 Control Plane for Elastic Networking . . . . . . . . . . . . . 40

3.5 Spectral Defragmentation . . . . . . . . . . . . 42

4 Architecture on Demand - Network Features 47

4.1 Scalability Analysis of AoD Based Networks . . . . . . . . . 47

4.2 Power Consumption Analysis of AoD Based Networks . . . . 49

4.3 Performance for Dynamic Scenario . . . . . . . . . . . . . 52

4.4 Survivability Analysis of AoD Based Networks . . . . . . . 54

$5 \quad$ Flexgrid Space Division Multiplexing (SDM) Networks $\quad 59$

5.1 Planning Strategy for Flexgrid SDM Networks with MCF . . 61

5.2 Designing Synthetic SDM Network with AoD Nodes . . . . . 62

6 Summary of Original Work $\quad 65$

6.1 Contributions of the Thesis . . . . . . . . . . . 65

6.2 Other Publications . . . . . . . . . . . . . . . 71

7 Conclusions and Future Work $\quad 73$

7.1 Conclusions . . . . . . . . . . . . . . . 73

7.2 Future Work . . . . . . . . . . . . . . 75

$\begin{array}{ll}\text { References } & 76\end{array}$

II Wavelength Switched Optical Networks 85

A Service Differentiated Provisioning in Dynamic WDM Networks Based on set-up Delay Tolerance 
B Fair Scheduling of Dynamically Provisioned WDM Connections with Differentiated Signal Quality

C Trading Quality of Transmission for Improved Blocking Performance in All-Optical Networks

III Elastic Optical Networks

D An Optimization Model for Dynamic Bulk Provisioning in Elastic Optical Networks

E Introducing Flexible and Synthetic Optical Networking: Planning and Operation based on Network Function Programmable ROADMs

F Dynamic Provisioning Utilizing Redundant Modules in Elastic Optical Networks Based on Architecture on Demand Nodes

G Routing, Spectrum and Core Allocation in Flexgrid SDM Networks with Multi-core Fibers

H Flexible and Synthetic SDM Networks with Multi-coreFibers Implemented by Programmable ROADMs 


\section{Part I}

\section{Introduction}





\section{Chapter 1}

\section{Introduction}

\subsection{Optical Core Networks}

Enormous developments have been made in optical networking over the past four decades. Starting from unrepeated point-to-point transmission, the inventions of optical amplifiers and wavelength division multiplexing (WDM) along with the innovation in fiber design have led to an explosion in system reach, system capacity, and network architecture. With advancements in the technology of optical components, the exploitation of optics has extended from WDM transmission to optical networking, which blends multiplexing, transmission, and optical switching. This has led to the building of the optical layer as a major part of the telecommunication transport infrastructure. The evolution in optical networking has focused on providing end user's demand for higher bandwidth in a cost-efficient manner.

In the last decade, progress in system capacity has slowed down due to saturation in fiber physical capacity while traffic demand has increased dramatically [1]. This phenomenon has diminished significantly the gap between system capacity and amount of traffic carried by the system. It has been forecasted that the network traffic will continue to rise owing to growth in number of Internet users along with the proliferation of fiber to the premises (FTTP) and other means of high bandwidth access. In addition, various new bandwidth intensive applications such as distribution of ultra-high definition TV (UHDTV), digital cinematic production, high definition interactive video conferencing, e-health, banking data backup storage, grid computing, e-science to mention a few, are expected to emerge over the next several years. These applications require diverse amount of bandwidth on-demand 
for relatively short duration; thus, the need for greater optical layer agility has grown. Considering the rapid exhaustion of the optical fiber physical capacity [2], new approaches that make the most out of the scarce network resources (fiber bandwidth) and fulfilling the future applications requirements need to be developed. With this in mind, this thesis focuses on some specific research issues in the context of dynamic WDM and flexible optical networks.

\subsubsection{Dynamic Optical Networks}

Modern core networks typically operate in the C-band portion of the spectrum with fixed grid of $50 \mathrm{GHz}$ defined by industry standard ITU G.694. Fibers with characteristics of single-core and single mode are employed. Network nodes are equipped with reconfigurable optical add/drop multiplexers (ROADM) to route wavelength connections optically, averting the expenses of optical-electronic-optical (OEO) conversion for connections that are traversed through the network node. Moreover, network capacity is expanded by enhancing wavelength bit rate from $10 \mathrm{Gbps}$ to 40 and $100 \mathrm{Gbps}$ by virtue of optical coherent technology. Although the networks are remotely reconfigurable through software control, the operations personnel generally initiate the provisioning process by using a planning tool. However, to accommodate future applications, the networks need to evolve into dynamic infrastructures, where connections can be rapidly established and torn down without the involvement of operations personnel. In such networks, the provisioning process is automatic and entirely under software control. The higher layers of the network routinely direct bandwidth request to the optical layer, which is then reconfigured accordingly. Connections may be provisioned and brought down in seconds, or possibly sub-seconds.

Dynamic networking is beneficial for both the network carriers and their customers, as it provides bandwidth where and when needed. Dynamic networking effectively diminishes the network bandwidth needs and allows a carrier to augment the revenues derived from a given level of deployed network capacity [3]. From the user perspective, bandwidth on-demand allows the user to set-up, tear down, and adjust connection bandwidth as needed. Dynamic service is more cost efficient than nailing up a maximum sized fixed connection. There is ongoing research to support set-up times on the order of $100 \mathrm{~ms}$ in the optical layer $[4,5]$, however, not all applications have such a stringent connection set-up requirement. For some applications, such as grid computing, e-science and cloud computing connection set-up times in the range of seconds to minutes would generally suffice. 
It is anticipated that dynamic networks will offer differentiated services to accommodate the different requirements of the various applications. Numerous potential service differentiation parameters are identified [6] which can constitute the service level agreement (SLA) for optical networks. Some of these parameters, for instance connection holding-time and connection setup time can be exploited in the context of resource optimization for dynamic connection provisioning. However, in a multiclass services scenario, there is a concern about whether network resources are used in a fair way, whereas less demanding classes have more chance to grab the resources. Thus, there is a need for provisioning strategies, which allocate the resources fairly among each service class. This matter and other related issues are investigated in Paper A, Paper B, and Paper $\mathbf{C}$ of this thesis in the context of dynamic WDM networks.

\subsubsection{Flexible Optical Networks}

To attain higher bit rate, e.g., $400 \mathrm{Gbps}$ or above over the current $50 \mathrm{GHz}$ fixed grid space WDM networks, more spectral efficient modulation schemes ( $8 \mathrm{bits} / \mathrm{s} / \mathrm{Hz}$ ) can be used theoretically. However, it is likely that such spacing would be technically challenging to acquire for rates beyond $100 \mathrm{Gbps}$ owing to the rapid decrease in the optical signal-to-noise ratio (OSNR). Rather it is anticipated that a bandwidth of 62.5 or $75 \mathrm{GHz}$ will be required for 400 Gbps technology [3]. Therefore, for a grid granularity of $50 \mathrm{GHz}$, it is essential to assign $100 \mathrm{GHz}$ for each $400 \mathrm{Gbps}$, thereby wasting 25-37.5\% of the spectral capacity. By providing the network with more flexibility, the fiber capacity can be utilized more efficiently, thereby extending the time until the capacity limit is accomplished. In this context, new optical transport networks, namely elastic optical networks (EON) [7] have been introduced as a solution to efficiently utilize the available spectrum resources. In the EON paradigm, the frequency spectrum is sliced into a number of small spectrum slices that are allocated to match as close as possible the spectrum requirement of each demand. As a result EON are able to show better spectrum utilization when compared to WDM networks. However, implementation of elastic networks necessitates innovation in network hardware and software, particularly a new type of ROADM is required that allows flexible spectrum to be switched from the input to the output ports. Among the different proposed models for flexible ROADM, the one based on architecture of demand (AoD) [8] provides a more scalable solution by means of its substantial flexibility. The modules (e.g., spectrum selective switches 
(SSSs), optical amplifiers) of an AoD are not hard-wired like in a static architecture, but can be connected together in an arbitrary manner and critically are decoupled from the input/output links. Component interconnections are provided by a high-port-count optical backplane, e.g., 3D-MEMS. This flexibility inherent in $\mathrm{AoD}$ can be exploited for different purposes, such as for cost-efficient, energy-efficient, and self-healing design of the nodes and networks. Moreover, for dynamic traffic, fast synthesis algorithms are essential to re-design the AoD ROADMs (and the network) on-the-fly in compliance to traffic requirements. Paper $\mathbf{E}$ and Paper $\mathbf{F}$ of this thesis analyze some of these aspects of AoD based flexible networks.

\subsubsection{Space Division Multiplexing}

The efficient utilization of spectrum resources through flexible networking takes relatively small steps towards alleviating capacity limits. This approach has a confined growth potential owing to the capacity crunch [2], on account of the finite transport capacity of a given single mode fiber core and the limited gain bandwidth of optical amplifiers [9]. Long-term solutions necessitate new technological developments on a similar scale to the WDM technology so that to keep up system capacity ahead of carried traffic demand cost-effectively. The nascent technology of Space division multiplexing (SDM) for high capacity transmission is such a solution with the scaling potential to handle the future traffic bandwidth requirements [10-12]. SDM can be realized by using multi-mode fiber (MMF), multi-core fiber (MCF), or few-mode multi-core fiber (FM-MCF). MMF employs the propagation of few independent modes within a single core. The number of modes supported by a fiber depends on the core size and the refractive index of the fiber. On the other hand, MCF has several cores embedded in the fiber cladding where each core acts as a single-mode fiber (SMF). The capacity potential of SDM has been manifested in several transmission experiments [11-14], exceeding $1 \mathrm{Pbps} /$ fiber. However, these solutions exhibit many implementation challenges and necessitate the development of new concepts, for instance, the spectrum and core allocation for connection demands. Paper G addresses the routing, spectrum and core allocation problem for designing flexible MCF based SDM networks. Similarly, Paper $\mathbf{H}$ extends the planning issue to synthetic and evolvable SDM networks implemented through programmable ROADMs. 


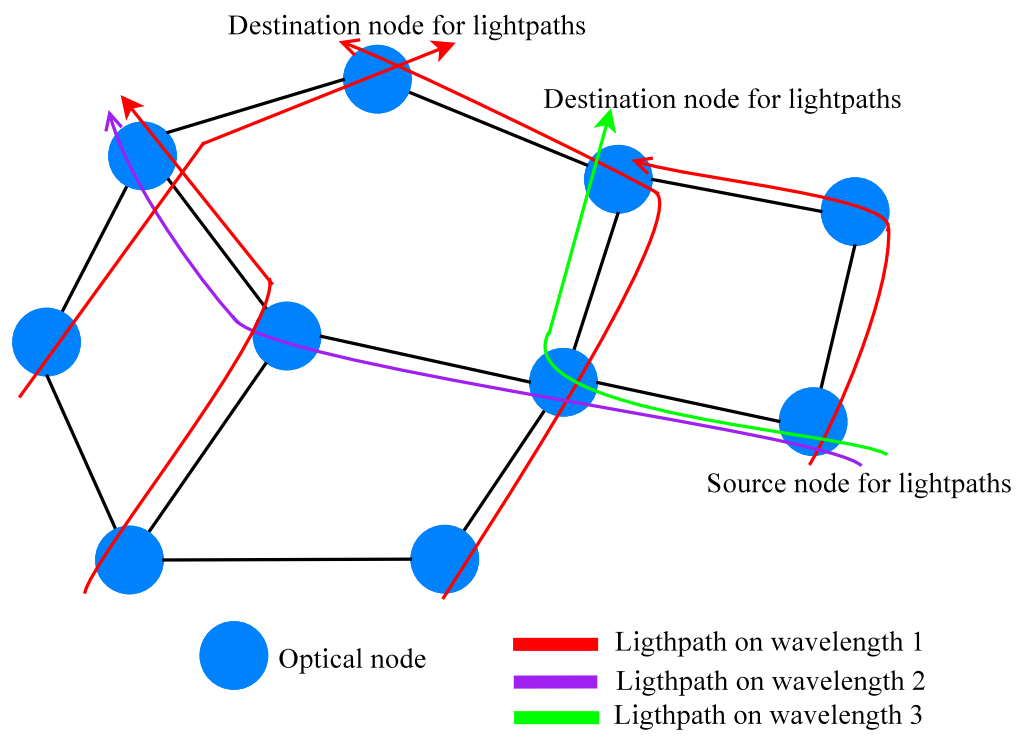

Figure 1.1: Lightpath configuration.

\subsection{Optical Switching}

Optical switching is the cornerstone in the implementation of optical networking. Like switching in the electrical domain, there are two main approaches for optical switching, namely, optical circuit switching (OCS) and optical packet switching (OPS). Also, in the recent years, optical burst switching (OBS) has been introduced as a compromise between OCS and OPS [15].

\subsubsection{Optical Circuit Switching}

In optical circuit switching (OCS), an end-to-end optical connection (called lightpath) that traverses multiple fiber links and optical nodes, e.g., ROADMs is established between a pair of source and destination nodes before the data is transferred as shown in Figure 1.1. The optical lightpath can be set-up with different levels of granularity, such as wavelength, waveband, or entire fiber, and are sometimes referred to as wavelength, waveband, and fiber switching, respectively. The OCS is the prevailing technique employed in core networks. With the introduction of swift reconfigurable ROADMs and fast tunable lasers in networks, dynamic on-demand OCS will become viable which will provide bandwidth on-the-fly to emerging bandwidth-on-demand applications. 


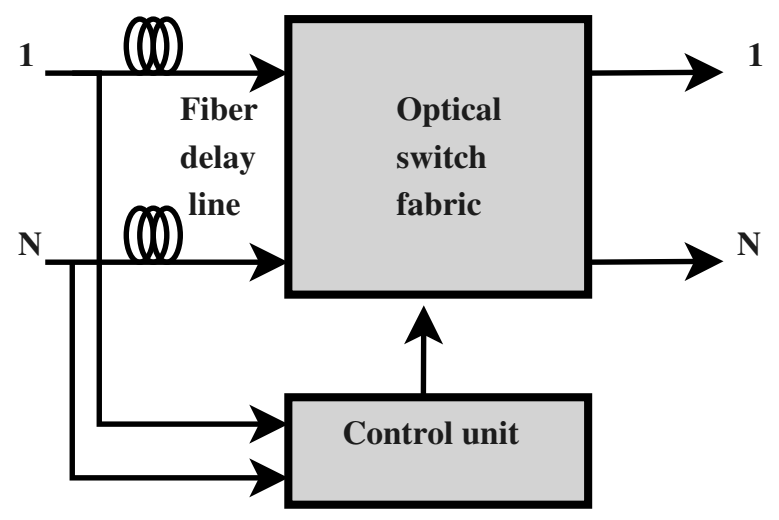

Figure 1.2: An optical packet-switch architecture.

The work of this thesis focuses on core networks, thus, employing OCS for connection provisioning. The granularity of OCS is varied from wavelength to waveband, as the work expands from WDM to flexible networks.

\subsubsection{Optical Packet Switching}

The "ultimate" optical network architecture proposed in literature is based on optical packet switching (OPS). An OPS is envisioned to provide the highest possible utilization of the optical core network. In OPS networks, data packets are switched and routed independently through the network entirely in the optical domain. An example of a basic optical packet-switched architecture is shown in Figure 1.2. A node contains an optical switch fabric, which is capable of reconfiguration on a packet-by-packet basis. The switch fabric is reconfigured based on the information contained within the header of a packet. The header itself is typically processed electronically, and can either be carried in-band with the packet or carried out-of-band on a separate control channel. Since it takes some time for the header to be processed and for the switch to be reconfigured, the packet may have to be delayed by sending it through an optical delay line. Although research in OPS is in progress and testbeds and demonstrators have emerged, the technology is still far from being mature enough for commercial deployment. Some technical obstacles must be overcome which include the development of very high speed (nanosecond) switching fabrics, optical buffers, header recognition, and optical clock recovery. 


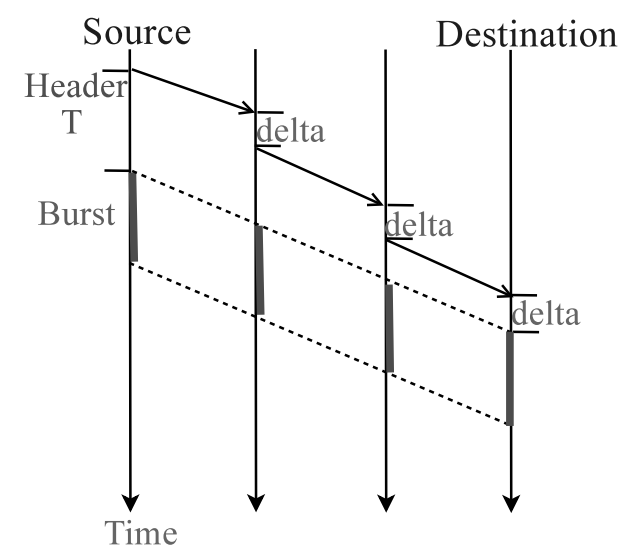

Figure 1.3: The use of offset time in OBS.

\subsubsection{Optical Burst Switching}

Optical burst switching (OBS) is designed to bridge the functional gap between OCS and OPS in the core network. In OBS network, a data burst consisting of multiple IP packets is switched through the network all-optically. A control packet is transmitted ahead of the burst in order to configure the switches along the burst's route. The offset time (Figure 1.3) allows for the control packet to be processed and the switch to be set-up before the burst arrives at the intermediate node; thus, no electronic or optical buffering is necessary at the intermediate nodes while the control packet is being processed. The control packet may also specify the duration of the burst in order to let the node know when it may configure its switch for the next arriving burst. By reserving resources only for a specified period of time rather than reserving resources for an indefinite period of time, the resources can be allocated in a more efficient manner and a higher degree of statistical multiplexing can be achieved. Thus, optical burst switching is able to overcome some of the limitations of static bandwidth allocation incurred by optical circuit switching. In addition, since data is transmitted in large bursts, optical burst switching reduces the requirement of fast optical switches that is necessary for OPS. Although OBS appears to offer advantages over OCS and OPS, several issues need to be considered before OBS can be deployed in working networks. In particular, these issues include burst assembly, signaling schemes, contention resolution, burst scheduling, and quality of service. 


\subsection{Methodology for Performance Evaluation of Optical Networks}

Experimental set-ups are often used by the research community for validation of hypotheses and the empirical results from the set-ups are analyzed to answer the aims or hypotheses. The selection of experimental set-ups depends on the research discipline. In the field of optical networking, researchers usually use discrete event driven simulation as a tool for evaluation of their proposed solutions. A brief description of discrete event driven simulation is presented below.

\subsubsection{Discrete Event Driven Simulation}

Simulation is the imitation of the operation of a real-world process or system over time. In optical networks, most of the time it is difficult to develop an analytical model for estimating system performance, due to complicated system structures and complex traffic patterns. Thus, discrete event driven simulation can be a feasible and efficient solution to assess network and system performance. In discrete event driven simulation a set of state variables collect all the information needed to define what is happening within a system. The system changes only at those discrete points in time at which events occur. The system state is updated at each event, along with occupying or freeing of system resources that might occur at that time.

In the framework of this thesis, a simulation tool based on discrete events has been developed for evaluation of dynamic scheduling algorithms. The developed simulator is specifically tailored to meet the requirements of each scenario under consideration. The arrival or departure of connection request acts as an event for the simulator, while the wavelengths on each fiber link of the network are considered as system resources. The system statistics, i.e., the parameters of interest (e.g., connections blocking percentage and resource utilization) are calculated from the system state variables. The simulation terminates after processing adequate number of events, while the number of runs of each simulation is set so as to achieve a desired confidence level.

\subsection{Organization of the Thesis}

This thesis focuses on service differentiated related research issues in dynamic WDM networks along with cost-efficient planning and operation strategies 
for flexible grid single-core and multi-core fiber optical networks.

The first part investigates the impact of quality of service differentiation by putting emphasis on time-based service level specifications such as setup delay tolerance and connection holding-time. This is further extended to incorporate the quality of the signal. The second part focuses on the planning and operation aspects for flexible networks implemented through traditional and AoD ROADMs.

The thesis is structured as follows. Chapter 2 provides an overview of the various topics underlying the WDM networks along with a summary of key results in the framework of dynamic WDM networks. Chapter 3 first discusses the various pieces that constitute elastic optical networking, and then presents the vital results in the context of dynamic bulk provisioning. Chapter 4 briefly presents the main findings of the analysis performed for the AoD based synthetic networks, while chapter 5 summarizes the key results for flexible multi-core fiber based SDM networks. A brief summary of each paper of the thesis work is provided in chapter 6 . Finally, chapter 7 concludes the research work presented in the thesis, along with identifying some interesting avenues for future research. 


\section{Chapter 2}

\section{Provisioning in Wavelength Routed Optical Networks}

Transparent WDM networks built on the concept of wavelength routing provide the backbone for the modern network infrastructure. Wavelength routing utilizes the concept of circuit switching to establish all-optical connections called lightpaths, which traverse multiple fiber links and optical nodes. A lightpath originates at an electro-optical $(\mathrm{E} / \mathrm{O})$ transmitter in the source node, stated as "added". It is assigned a wavelength on each traversed link, and is optically switched at the intermediate nodes. The lightpath terminates at an optical-electro $(\mathrm{O} / \mathrm{E})$ receiver in the destination node, where it is said to be "dropped". Moreover, a lightpath is analogous to an electrical circuit, which must be requested, provisioned, and when it is no longer required, torn down. Optical add/drop multiplexers (OADMs) are the specialized equipment employed for the realization of the add, drop, and optical switching of the lightpaths. The reconfigurable-OADM (ROADM) is a more agile form of OADM, where the optical switching functionalities are performed by active optical devices, governed by a coupled control and management plane. As a result, lightpaths configuration can be software-driven without any on-site manual intervention. This feature reduces the operational cost of the network and is a step forward towards dynamic networks.

Figure 2.1 shows a ROADM architecture with nodal degree three (i.e., connected to three other nodes), that relies on the broadcast-and-select scheme to implement the switching functionality. The optical signals received at any specific input port is broadcast to the other output ports and to the 


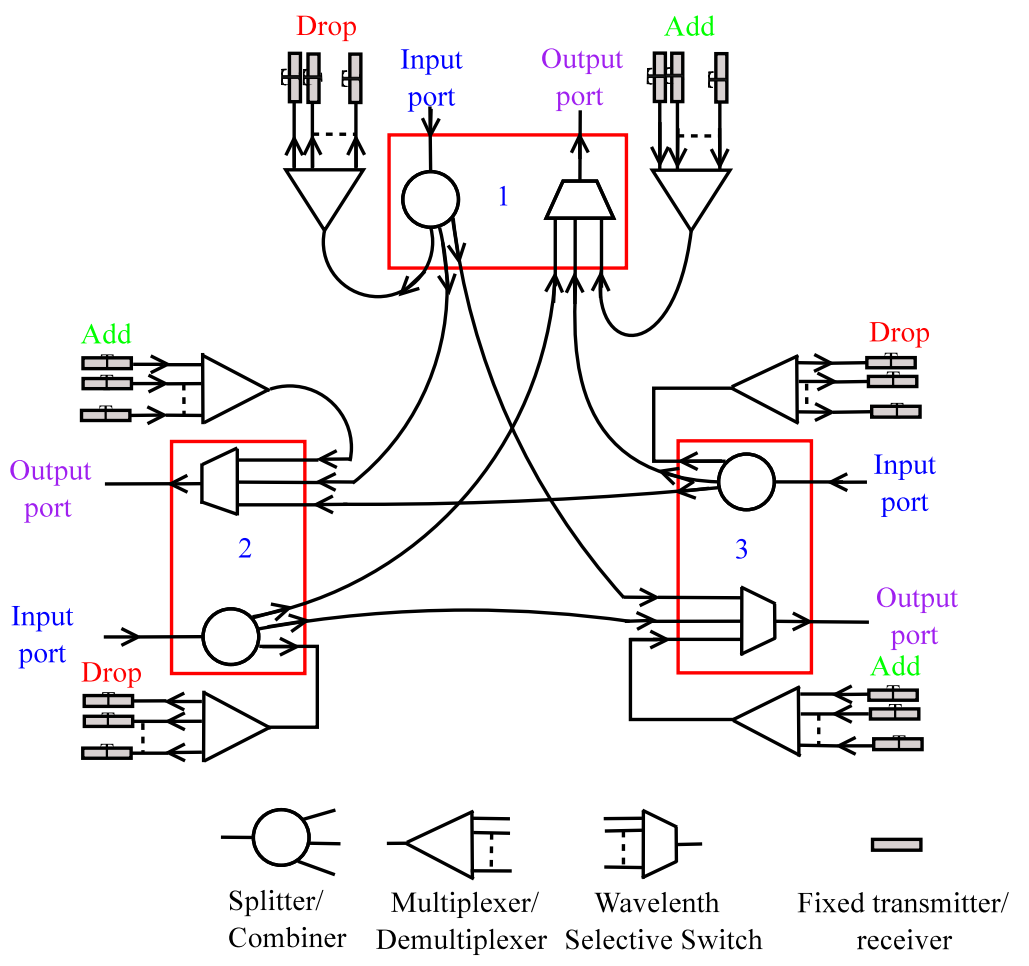

Figure 2.1: Broadcast-and-select ROADM architecture [16].

drop module. The drop module employs a passive demultiplexer to separate the WDM connections into different drop ports where the transceivers are placed. Moreover, the wavelength selective switches (WSSs) deployed at the output ports combine the optical signals delivered from the other directions and the add module. The WSS is a reconfigurable device which can switch any wavelength received from any direction to its output port. Finally, the add module uses the multiplexer to inject the composited signal of the transceivers into an input of the WSS.

A lightpath is realized by finding a path between the source and the destination and assigning a free wavelength on all the links of the path. The selection of the path and the wavelength to be employed by a lightpath is an important optimization problem, known as the routing and wavelength assignment (RWA) problem. Setting up a lightpath for a connection request by using the RWA technique is referred to as connection provisioning [17]. The remainder of this chapter presents a review of the various issues related to transparent WDM networks, and a brief description of the thesis contribution in the framework of dynamic WDM networks. 


\subsection{The RWA Problem}

The performance of a network depends not only on its physical resources (e.g., number of wavelengths per fiber, fiber links, etc.) but also on how it is controlled. The objective of an RWA algorithm is to achieve the best possible performance within the limits of the physical constraints. The constraints of the RWA problem may include wavelength continuity, distinct wavelength, physical layer impairments (PLI), and traffic engineering considerations. The wavelength continuity constraint requires a connection to use the same wavelength along a lightpath. The wavelength continuity constraint can be relaxed by deploying wavelength converters in the network nodes. The distinct wavelength constraint imposes that all lightpaths traversing through the same link (fiber) must be allocated different wavelengths. The PLI constraint concerns how to select a wavelength and/or path that guarantee the required level of signal quality. The traffic engineering constraint aims to improve resource-usage efficiency and reduce connection blocking probability.

Initial studies on RWA problems relaxed the PLI constraint by considering a perfect transmission medium, and assumed all outcomes of the RWA algorithms to be valid and feasible. However, the optical signals propagating through the fiber links and passive and/or active optical components encounter different sort of impairments that affect the signal intensity level, as well as its temporal, spectral and polarization properties. Thus the actual performance of the system may be unacceptable for some of the lightpaths. This thesis studies RWA algorithms with only wavelength continuity constraint in Paper A. For Paper B and Paper C, RWA algorithms with both wavelength continuity and PLI constraints are employed. A brief description of the classification of PLI, traffic models, approach for solving RWA problem, and RWA algorithms for ideal and non-ideal physical layer are presented in the following subsections.

\subsubsection{Physical Layer Impairments}

The physical layer impairments encountered in optical networks can be classified into two categories: linear and non-linear impairments. Linear impairments affect each wavelength (optical channel) individually without creating interference or disturbance among the wavelengths, and are independent of the signal power. Nonlinear impairments, which are signal power dependent not only affect each wavelength, but also cause interference between them. 
The prominent linear impairments are: fiber attenuation, component insertion loss, amplified spontaneous emission (ASE) noise, cross-saturation of amplifier, chromatic dispersion (CD), polarization mode dispersion (PMD), polarization dependent losses (PDL), crosstalk, and filter concatenation. ASE noise is generated by optical amplifiers used to compensate the optical power losses due to fiber attenuation. CD incurs when the constituent frequencies of the optical signal propagate with distinct velocities thus reaching the destination at different times. PMD is a pulse broadening phenomenon originating from the fact that the orthogonally polarized components of a pulse travel along the fiber with different group velocities due to fiber birefringence. Similarly, the important non-linear impairments can be summarized as self phase modulation (SPM), cross phase modulation (XPM), four wave mixing (FWM), stimulated brillouin scattering (SBS), and stimulated Raman scattering (SRS). SPM occurs due to the fiber refractive index dependence on the signal intensity while XPM is inflicted on an optical channel by the intensity of the other signals co-propagating in the same fiber. FWM is the phenomenon that generates new optical signals by mixing three optical channels co-propagating simultaneously in the fiber. Similarly, SBS incurs when an optical signal in the fiber interacts with the density variations, such as, acoustic phonons and changes its path. Finally, the SRS effect causes optical signal power from lower wavelength channels to be transferred to the higher wavelength channels, when two or more optical signals at different wavelengths are injected into a fiber.

A detailed description of all these impairments, their effect on optical feasibility, and techniques to mitigate their impact can be found in $[18,19]$. Note that none of the existing studies has considered all the PLI in the RWA process. Each RWA algorithm incorporates only some specific PLI that depend on the assumed network scenario. The PLI considered in this work are: crosstalk, ASE noise, cross-saturation of erbium-doped fiber amplifier (EDFA), receiver noise, fiber attenuation, and power loss in the optical components.

\subsubsection{Traffic Models}

Two alternative traffic models are considered for all-optical networks: static traffic and dynamic traffic. Figure 2.2 presents a conceptual view of a general static/dynamic traffic model. For static traffic, a set of connection requests is known in advance. The objective of the RWA problem for static traffic is to establish a set of light paths to accommodate all the connection requests while minimizing the number of wavelengths used in the network. 


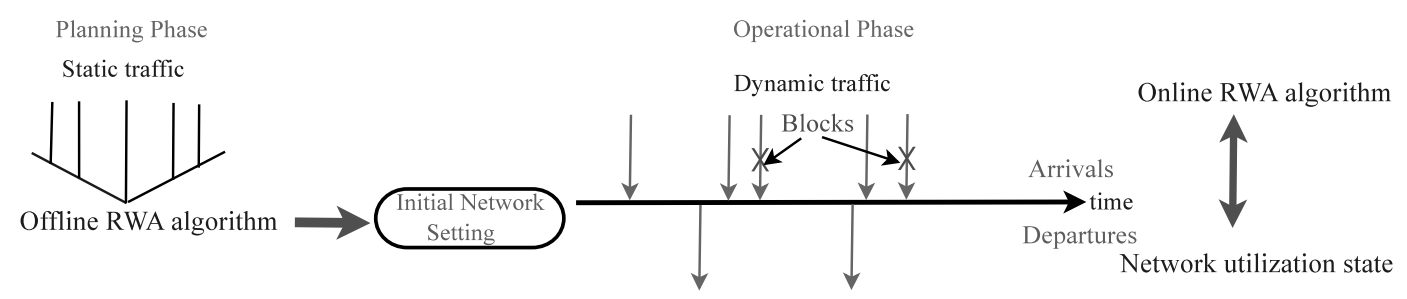

Figure 2.2: General traffic model.

The static RWA problem arises naturally in the design and capacity planning of an optical network (offline RWA algorithms). For dynamic traffic, connection requests arrive to and depart from the network dynamically in a random manner. A lightpath is set-up when there is a connection request and is released when the data transfer is completed. The goal of the RWA problem for dynamic traffic is to route and assign wavelengths in such a way as to minimize the blocking probability of the network (online RWA algorithms). A connection is said to be blocked if there are not enough resources available to establish it. The dynamic RWA problem is encountered during the real-time network operational phase of the optical network. This work investigates the operational performance of WDM networks, thus the dynamic traffic model is adopted for the RWA problem.

\subsubsection{Approaches for Solving the RWA Problem}

The RWA problem for static traffic (i.e., offline RWA) is known to be NPcomplete, which means that finding the optimal solution in polynomial time is not achievable. Furthermore, for the dynamic traffic case where the entire traffic demand set is unknown in advance, it not possible to compute an optimal solution for the RWA problem. Solving the RWA problem requires the solution of two sub-problems namely, the routing and wavelength assignment sub-problems. These two sub-problems can be addressed either jointly or separately by using one-step or two-step approach, respectively. The two-step approach, which first solves the routing and then the wavelength assignment sub-problem is suboptimal but less complex.

For static traffic, connection requests are assumed to be established for relatively long duration and time for solving RWA is not a critical issue. Therefore, it is reasonable to optimize the allocation of network resources by finding an optimal solution through the one-step approach. However, 
in the dynamic traffic case, connection requests must be set-up promptly upon arrival for comparatively smaller time periods. Thus, it is more practical to solve routing and wavelength assignment as sub-problems in order to set-up the connection requests swiftly. This study examines dynamic traffic scenarios and hence utilize the two-step approach for addressing the RWA problem.

\subsubsection{RWA Algorithms for Ideal Physical Layer}

The routing algorithms can be divided into two categories: static and adaptive algorithms. In static routing algorithms (e.g., fixed routing [20], fixedalternative routing [20]), one or several paths are computed independently of the network state for each source-destination pair. Such algorithms are executed offline and the computed paths are stored for later use, resulting in low latency during connection provisioning. However, these paths cannot respond to dynamic traffic conditions. Adaptive routing algorithms (e.g., shortest-path [20], shortest-cost-path [21], and least-congested-path [22]) are executed at the time a connection request arrives and require network nodes to exchange information regarding the network state. This exchange of information may increase the connection set-up time and computation cost, but in general, adaptive algorithms improve network performance.

Similarly, there are numerous wavelength-assignment algorithms reported in literature for both single and multi-fiber networks. For single-fiber networks, the Random algorithm [20] selects randomly one free wavelength from the unused wavelengths on the chosen path. The First-Fit algorithm [20] picks the free wavelength with the smallest index. In the Most-Used algorithm [20], the free wavelength which is used most often in the network is selected. In the Least-Used algorithm [20], the free wavelength which is used least in the network is selected. All these algorithms that are proposed for single fiber networks can also be extended to multi-fiber networks with and without modification [23]. However, other algorithms, e.g., Min-Product [24], LeastLoaded [25], Max-Sum [26], and Relative-Capacity-Loss [27] have been proposed for multi-fiber networks to further improve the network performance.

Random and First-Fit are the simplest algorithms in terms of computational complexity, and their running times are on the order of $O(W)$, where $W$ denotes the number of wavelengths in the network. Least-Used and Most-Used are more complex than Random and First-Fit. For a single-fiber network with $W$ wavelengths and $L$ links, Least-Used and Most-Used will run in $O(W L)$ time [21] while for multi-fiber network with $M$ fibers on each link, 


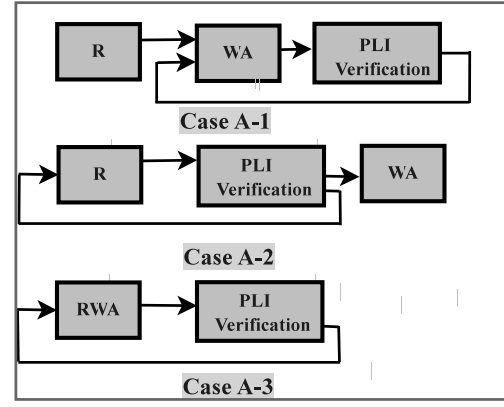

R: Routing decision WA: Wavelength Assignment RWA: Routing and Wavelength Assignment PLI: Physical Layer Impairments
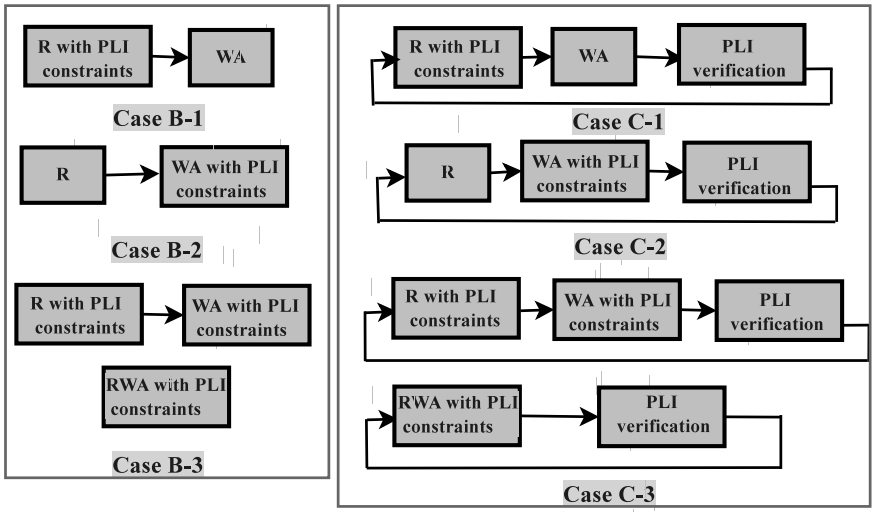

Figure 2.3: Various PLI-RWA approaches [28].

these algorithms will run in $O(W L M)$ time [21]. The computations in MinProduct and Least-Loaded will take $O(K W)$ time, where $K$ denotes the number of network nodes. Finally, Max-Sum and Relative-Capacity-Loss are relatively expensive as for worse case scenario their computation cost will be $O\left(W K^{3}\right)[21]$.

Paper A investigates single-fiber networks, and utilizes static routing and First-Fit wavelength assignment algorithms due to their low computational time and network overhead.

\subsubsection{RWA Algorithms for Non-Ideal Physical Layer}

The incorporation of PLI in transparent optical network planning and operation has recently received attention from the research community. The PLI incurred due to the non-ideal network components reduce the number of candidate paths that can be selected for routing. Furthermore, a wavelength selection made for one lightpath affects and is influenced by the wavelength choice made for the other lightpaths, due to the PLI effect. For the incorporation of PLI in the RWA algorithms three approaches have been adopted in the literature: (a) calculate the route and the wavelength in the traditional way, i.e., as described above for the ideal physical layer, and verify the feasibility of selected lightpath by considering the PLI; (b) incorporate the PLI values while selecting the route and/or wavelength for a connection request: (c) consider the PLI values in the routing and/or wavelength assignment decision and finally verify the signal quality of the candidate lightpath. 
These approaches and their various combinations are depicted in Figure 2.3 (adapted from [28]).

In the A-1 case the route and wavelength are computed without considering the PLI constraints, but the wavelength assignment (WA) decision can be modified after the verification phase. A-2 calculates the route without taking into account the PLI, but there is an option of recomputing the route if the PLI constraints are not fulfilled by the candidate route(s). Similarly, A-3 computes the route and wavelength (either in one step or two) using traditional schemes and then checks the PLI constraints in order to possibly change the RWA decision. The approaches in group B address the RWA problem by incorporating the physical layer information: in the B-1 case the route is computed using PLI constraints: in the B-2 case these constraints are considered in the wavelength assignment process; lastly in the B-3 case the PLI constraints are taken into account in both route and wavelength selection. Some of the works that adopt the last approach use the physical layer information as weights associated with the links, in order to calculate the minimum cost lightpath. Approach $\mathrm{C}$ is the combination of the last two approaches. The PLI constraints are taken into account in the routing $(\mathrm{C}-1)$, or in the wavelength assignment $(\mathrm{C}-2)$ or in both (C-3); but the PLI constraints are finally verified that enable the re-attempt process in the lightpath selection phase.

Similarly, there are two procedures for accounting for the interference among the lightpaths while addressing the PLI-RWA problem [29]. The first technique chooses a lightpath for a new connection request that has acceptable transmission quality under a worst case interference assumption, ensuring that the selected lightpath will not become infeasible due to the possible establishment of future interfering connections. The second technique considers the current network state and the actual interference among the lightpaths. This technique performs a cross layer optimization between the network and physical layers, and checks whether or not the establishment of the new lightpath will make any already established connection infeasible. The former approach is less complex and calculates a quick and stable lightpath at the expense of reducing the candidate path space available for routing. The latter approach performs better by exploring a larger path space at the cost of adding complexity introduced by the cross-layer optimization.

This thesis work picks the C-2 approach for the part that takes into account the PLI (Paper B, Paper C), and employs the current network state to calculate the actual interference among the lightpaths. In this way, more connection requests will be set-up in the network and the available network 
resources will be optimally utilized.

\subsection{Optical Network Control and Management}

Network control and management is a general term used for a set of functionalities that include network topology discovery, dissemination of network state information, neighborhood discovery, fault management, and performance monitoring operations. These functionalities are mainly achieved through two protocols namely: protocol for topology and network state information dissemination and protocol for signaling. Furthermore, the set is extended to cover connection management operations (i.e., setting-up, preservation, and tear down) of the requests in a dynamic scenario.

\subsubsection{Control Architecture for RWA}

Connection requests are set-up in the network after providing resources (wavelength) by solving the RWA problem. Two frameworks, i.e., centralized and distributed [20] control have been proposed for the network provisioning operations. The centralized control architecture adopts a similar technique as used in a circuit-switched telephone network, whereas, the distributed control architecture resembles the approach implemented in packet data networks such as the Internet. In the centralized architecture, a controlling node monitors the network state and controls all resource allocations. When a connection request is received, an edge node sends a message to the controlling node. The controlling node executes the routing algorithm and the wavelength-assignment algorithm. Once a path and a free wavelength are selected, the controlling node will reserve resources (i.e., choose wavelength) on all nodes along that path. The distributed control architecture broadcasts information about the network state periodically, which enables each edge node to compute the path upon getting a connection request. When receiving a connection request, an edge node first executes the routing algorithm to compute a path and then it starts the wavelength-reservation protocol. The wavelength-assignment algorithm can be executed either by the destination node or by the source node to pick a free wavelength.

The Internet engineering task force (IETF) has defined a path computation element (PCE) based framework [30], where the path computation and resource allocation functions are performed by a single location in the network. The benefit of centralizing all path computation and resource allocation in 
one entity is the potential for optimality. The PCE can compute the best path and wavelength for a new connection request by exploiting the full knowledge about the network. In addition, the PCE can operate in a batch mode, where resource computation are performed for a set of requests rather than processing each request one by one. This potentially enhances the network provisioning capability [31].

\subsubsection{Standards for Control Plane}

For automated provisioning of connection requests in optical networks, there are two dominant standards. ASON (Automatically Switched Optical Network) standardized by ITU-T partitions the network into three layers: the transport plane, the control plane, and the management plane. The transport plane serves to transfer user information from source to destination in the optical domain along a lightpath. The control plane plays a central role and supports the functionalities of managing and allocating network resources, signaling the creation of a lightpath, providing network-to-network interfaces (NNI) to facilitate the exchange of relevant data with neighboring domains. Moreover, it also provides user to network interfaces (UNI) to enable automated bandwidth provisioning on demand. The management plane is responsible for managing the control plane and can be either centralized or distributed depending on the network. The responsibilities of management plane include configuration management of the control plane resources, and transport resource in control plane.

The IETF has proposed the generalized multi-protocol label switching (GMPLS), which is derived from multi-protocol label switching (MPLS). MPLS is basically a technique that allows traffic engineering by creating virtual switched circuits through an IP network. GMPLS extends this approach from packet switching to cover circuit-oriented optical switching technologies such as time division multiplexing (TDM), and WDM. Apart from control plane architecture definitions, GMPLS also defines a suite of protocols which can be used on transport network architectures based on either the ASON overlay network or its own GMPLS overlay [32]. In other words, GMPLS provides a set of protocols for the implementation of the tasks abstractly defined in ASON.

The GMPLS protocol suite includes link management protocol (LMP), open shortest path first with traffic engineering extensions (OSPF-TE), and resource reservation protocol with traffic engineering extensions (RSVT-TE). 


\begin{tabular}{|c|c|c|c|c|c|}
\hline \multicolumn{6}{|c|}{ Optical SLA } \\
\hline \multicolumn{5}{|c|}{ Optical SLS } & \\
\hline \multicolumn{3}{|c|}{ Service Boundary } & \multicolumn{2}{|c|}{ Flow Identifier } & \\
\hline \multicolumn{3}{|c|}{ Traffic Conformance } & $\mathrm{Ce} \mathrm{Tr}$ & hroughput & \\
\hline \multicolumn{3}{|c|}{ Service Schedule } & & $\begin{array}{l}\text { mnection } \\
\text {-up Time }\end{array}$ & (1) \\
\hline \multicolumn{5}{|c|}{\begin{tabular}{|l|l|l|} 
Routing & Route & Excess \\
\end{tabular}} & 60 \\
\hline \multicolumn{2}{|c|}{\begin{tabular}{|l|} 
Service \\
Performance \\
Guarantees \\
\end{tabular}} & \multicolumn{3}{|c|}{\begin{tabular}{l|l|} 
Service & Mean \\
Availability & Down \\
and Resilience & Time \\
\end{tabular}} & \\
\hline Delay & \multicolumn{2}{|c|}{\begin{tabular}{c|} 
Recovery \\
Time
\end{tabular}} & \multicolumn{2}{|c|}{ Confidentiality } & \\
\hline \multicolumn{4}{|c|}{ Out-of-service Criterion } & Distance & \\
\hline \multicolumn{5}{|c|}{$\begin{array}{c}\text { Recovery Time w/Degraded } \\
\text { Performance }\end{array}$} & \\
\hline
\end{tabular}

Figure 2.4: Parameters for optical SLA [6].

In addition, intermediate system to intermediate system (IS-IS) and constraintbased routing label distribution protocol (CR-LDP) can be used as alternative for routing protocol and resources reservation protocol, respectively.

\subsection{Differentiated Classes of Service in Optical Net- works}

The dynamic optical networks will provide a unified platform for existing and new emerging network applications. It is expected that the networks will not only fulfill the bandwidth requirements of these diverse applications, but also guarantee appropriate quality conforming to customers needs. The quality of a connection may be examined from three aspects, namely, quality of service (QoS), grade of service (GoS), and quality of protection (QoP) [33]. QoS covers the connection parameters that affect data flow after the lightpath is set-up in the network. GoS comprises all parameters relevant to the connection set-up phase. Finally, QoP describes all features of the connection related to protection and restoration. In the context of these quality aspects, the creation of specifications and standards become mandatory, yielding to an optical service level agreement. 
A service level agreement (SLA) is a formal contract between a service provider and a customer that contains detailed technical specifications called service level specifications (SLSs). The SLS consists of a selected set of QoS, GoS, and QoP parameters and their values that together define the service offered to a customer. There are no standards defined for the contents of an optical SLA, however Figure 2.4 depicts some parameters that can be included in an optical SLA as proposed in [6]. The most prominent GoS parameters are the connection set-up time and connection blocking probability. The connection set-up time indicates the time required from the instant a connection request is generated to the moment the connection is completely established and ready for data transfer. Similarly, quality of transmission for optical signal is one of the most important QoS parameter.

Service differentiation is a valuable opportunity for operators to maximize the revenues derived from their infrastructure, by selling high added-value services. Different classes of services, namely, premium, gold, silver, and bronze with different specifications for QoS, GoS, and QoP parameters have also been proposed in [6]. Differentiated services will not only assist the customers to select an appropriate service class in compliance with their needs, but will also benefit the service providers by managing their network resources efficiently.

This thesis work looks upon service differentiation issues in dynamic optical networks, and classifies the connection requests into different service classes based on their set-up delay tolerance [34] and signal quality of transmission requirements (Paper A, Paper B). More demanding service classes generally undergo high blocking. However, the proposed strategies for service differentiation are particularly tailored to facilitate the provisioning of the most stringent service class so as to balance the success rate among the different classes. Furthermore, the strategies make use of the connections holding-time awareness [35] for enhancing their performance.

\subsection{Time Dimension Issues for Connections Pro- visioning}

Network connections provisioning and consequently network blocking performance can be improved significantly by exploiting the temporal related metrics of SLA, such as connection set-up time and connection holdingtime. Similarly, reserving network resources prior to utilizing them can also enhance network connections provisioning. 


\subsubsection{Scheduled or Advance Reservation}

The RWA models explained above only consider the so-called immediate reservation (IR) requests, which are made just at the time when the network resources (i.e., wavelength) are actually required. A connection request will be blocked if the network resources are not available at the time when they are needed. IR benefits those applications that are delay-sensitive, for instance online gaming and video streaming. However, some applications, for instance banking backup data, and grid computing can be delay-tolerant as long as the network resources are provisioned before a deadline. For such applications, an advance reservation (AR) [36] or scheduled mechanism can greatly enhance their provisioning probability. Unlike immediate reservation, an AR request specifies a start time that is in the future and a specific holding-time. The time interval between reservation and utilization of the network resources is denoted as the book-ahead time. AR not only provides guaranteed services to network users, but also allows network operators to plan their resources allocations efficiently, and consequently increases their revenue. Several models for AR based on whether the starting time and/or holding-time are specified or not, are described in [36]. All these models have been thoroughly investigated by the research community, and a comprehensive literature survey on AR RWA can be found in [37]. An important design decision for $\mathrm{AR}$ is whether the scheduling function should be centralized or distributed. There are three main aspects of the design process: choosing a start time (for requests with flexible starting time), selecting a route, and allocating wavelengths. These processes are well suited to centralized operation, where batch scheduling can be executed to improve optimality, or where the reserved resources can be re-optimized $[38,39]$ for scheduled connections that are not yet in service. Scheduling is more challenging in a distributed set-up as it requires information of the resources that have already been reserved while accommodating a new AR connection request. This would lead to flooding of information regarding all accepted scheduled connection requests to each of the network nodes.

\subsubsection{Holding-time}

Holding-time describes the duration for which a connection remains active and occupies the network resources. As mentioned above, new applications are emerging with requirements of large bandwidth over relatively short and predictable periods of time, for instance video distribution of important sport or social events. It is expected that the holding-time of connection 
requests can be known in advance, as it is considered to be a metric of the SLA between the network operator and its customers [40]. The prior knowledge of connections holding-time has been exploited for different dynamic network scenarios, for instance efficient utilization of lightpath capacity and bandwidth allocation for the traffic grooming problem [35], enhancement of backup resource shareability in survivable networks [41] and for efficient load balancing [42].

This thesis also exploits the holding-time awareness by combining it with set-up delay tolerance, in the context of resource optimization for dynamic lightpath provisioning. The knowledge of connections holding-time can be utilized to retrieve the information about the departing connections, and that information can be used for efficient provisioning of waiting connection requests. The performance improvement due to the combination of these two metrics is investigated with and without the assumption of an ideal optical medium.

\subsubsection{Set-up Delay Tolerance}

Advance reservation (AR) enables network users to ensure the availability of resources prior to when they are required. For immediate reservation (IR) connection requests, which require immediate provisioning and allocation of resources it may happen that the resources are not free at the time when the request arrives. However, they might be free shortly thereafter when some active connections depart from the network. For IR connection requests, set-up delay tolerance [34] has been proposed for improving the connections provisioning. Set-up delay tolerance describes customer patience, i.e., the maximum duration a customer is willing to wait until the connection is setup. Set-up delay tolerance is a special case of AR tailored to facilitate the provisioning of delay-sensitive connection requests. The waiting time in the delay tolerance case is expected to be a fraction of the connection request holding-time for most of the applications, and the connection request is provisioned immediately after the required resources become free within its waiting time, which leads to efficient utilization of resources.

Paper A looks into a scenario where connection requests are categorized into three service classes (i.e., SC-I, SC-II, and SC-III) based on the set-up delay tolerance. Among these SCs, SC-III has high set-up delay tolerance which is followed by SC-II, and then by SC-I. It is observed that SC-II and SC-III performance is improved compared to the basic approach, i.e., provisioning with no delay tolerance (NDT) as shown in Figure 2.5. However, 


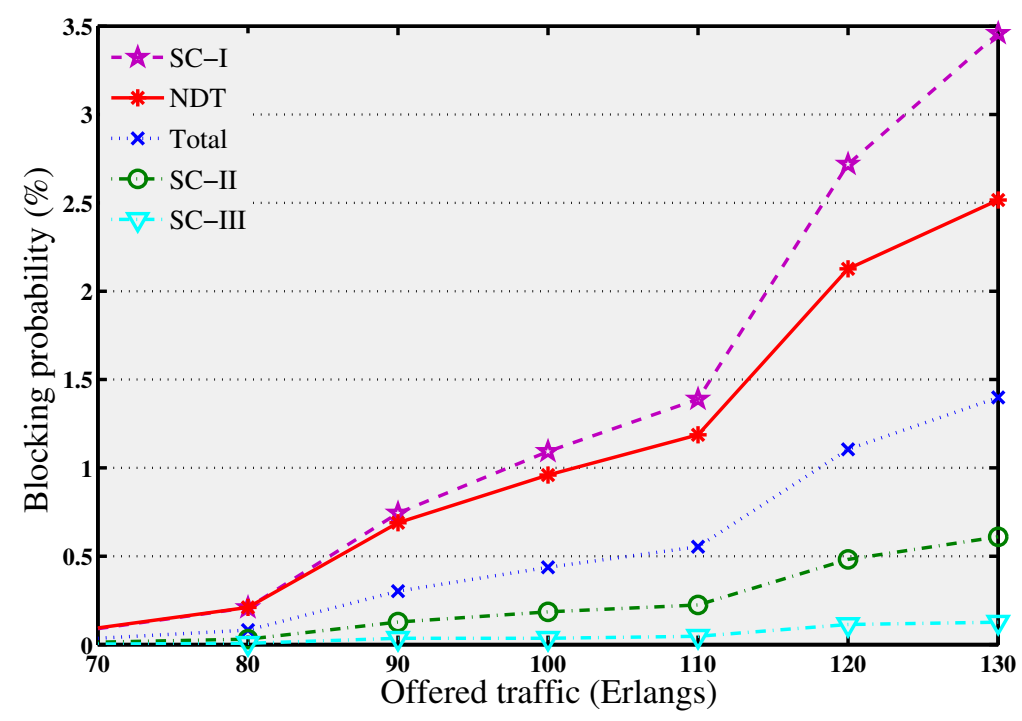

Figure 2.5: $\quad$ Blocking probability for 30 nodes European topology.

the blocking performance of SC-I, which requires stringent set-up delay tolerance, deteriorates compared to NDT. High set-up delay tolerance of SC-II and SC-III enabled these SCs to occupy the network resources, which consequently reduce the chance for SC-I connection requests to get connected. Paper A proposes different scheduling strategies for promoting the requests belonging to smaller set-up delay tolerance SCs, such as giving priority in the rescheduling queue, reserving some fraction of available resources, and augmenting the research space by providing some extra paths. Moreover, the impact of network nodal degree, load, and network traffic distribution among the SCs on the performance of the proposed strategies is also investigated.

Among the proposed strategies in Paper A, the ones based on the reservation of a fraction of available resources and extra paths along with priority in the rescheduling queue are successful in eliminating the adverse effect of high delay tolerant classes on the BP performance of the SC with low delay tolerance. These strategies can be used even without the queue priority for the low set-up delay tolerance SC, but at the expense of enhancing the reserved resources and extra path sets. The strategy of giving priority only to low delay tolerant class requests in the rescheduling queue can reduce this adverse effect, although it cannot overcome the degradation completely. 


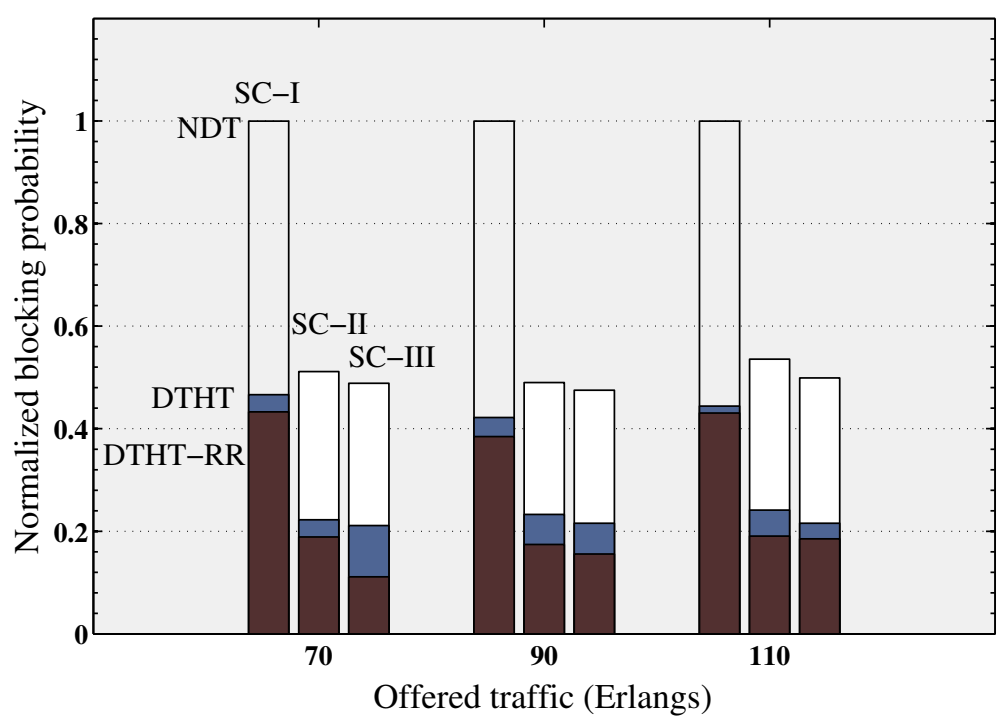

Figure 2.6: Normalized blocking probability for 14 nodes NSF topology scaled down by a factor of 10 .

\subsection{Quality of Transmission}

The quality of transmission (QoT) of the provisioned connections (lightpaths) can be measured in terms of bit error rate (BER) or equivalently, in terms of Q-factor [18]. The BER of an optical path depends on the span of the physical links and the number and type of links/nodes traversed by the optical signal. At low line rates, i.e., $2.5 \mathrm{~Gb} / \mathrm{s}$ and $10 \mathrm{~Gb} / \mathrm{s}$ BER measurements take considerable amount of time to achieve statistically valid results [19]. However, the Q-factor which measures the quality of an analog transmission signal in terms of its signal-to-noise ratio (SNR) can determine error ratio faster than the BER test. A high Q-factor means better optical signal-to-noise ratio (OSNR), and thus lower probability of bit errors. The QoT requirements varies with applications, for instance sensitive backup data (e.g., financial transactions and banking) demands a BER value of $10^{-15}$ or equivalently a Q-factor equal to 9, compared to Gigabit Ethernet which requires these values to be $10^{-12}$ and 8 , respectively [19]. Forward error correction (FEC) reduces the bit errors, however applications with tight latency requirements (e.g., interactive communication and streaming data) might have less room for error correction and consequently require high QoT [43].

When exploring a scenario where connection requests are classified in different service classes based on their QoT requirements, the provisioning success 
rate might become unbalanced towards those connection requests with less stringent requirements. I.e., not all the connection requests are treated fairly. To examine such a situation, Paper B considers the differentiation of service classes in terms of signal quality, where each service class, namely, SC-I, SC-II, and SC-III, requires a different QoT level, i.e., SC-I demands higher QoT followed by SC-II, and then by SC-III. Figure 2.6 shows the normalized $\mathrm{BP}$ for two strategies that exploit set-up delay tolerance and holding-time awareness with advance reservation of network resources (DTHT-RR) and without reservation (DTHT). These strategies are compared with a basic approach, i.e., the impairment-aware provisioning with no delay tolerance (NDT). The BP is normalized using the SC-I BP value for NDT. The BP of each class and the total network $\mathrm{BP}$ are reduced significantly when a set-up delay tolerance and a holding-time aware approach is used. However, the BP of SC-I is $48 \%$ higher than SC-II for NDT, and this difference becomes more substantial when the DTHT and DTHT-RR approaches are used, i.e., $50 \%$ and $53 \%$, respectively. In order to balance the provisioning success rate among all service classes and to improve the fairness of the network, Paper B proposes three fair scheduling algorithms in a dynamic traffic scenario. Each one combines in a different way the concept of both set-up delay tolerance and connection holding-time awareness, and are able to guarantee a fair treatment reaching up to $99 \%$ in terms of Jain's fairness index, considering the per-class success rate.

One of the reasons for connection requests blocking in a dynamic traffic environment is the difficulty to provision a connection with the requested QoT level regardless of the wavelength resources availability. However, in dynamic networks the optical impairments (specifically the traffic dependent impairments e.g., crosstalk) vary with time. Connection requests that are unable to attain the targeted QoT level at their time of arrival might have that level for most of their holding-time. Similarly, the disturbed QoT target of an existing lightpath (because of the crosstalk created by new provisioned connection) might recover after a while, when the crosstalk level reduces due to connections departure. In addition, there are some applications which may tolerate momentarily degradation in QoT [6]. For instance, delay intolerant applications, such as interactive video can tolerate a certain amount of packet loss without the resulting impairments becoming significantly annoying to the user [44]. For such applications, Paper C proposes a QoT trading strategy, which exploits the possibility to allow a tolerable signal quality degradation during a small fraction of the holding-time for connection provisioning. Two new customer specified service performance parameters, namely, tolerable quality level and degraded quality time are de- 


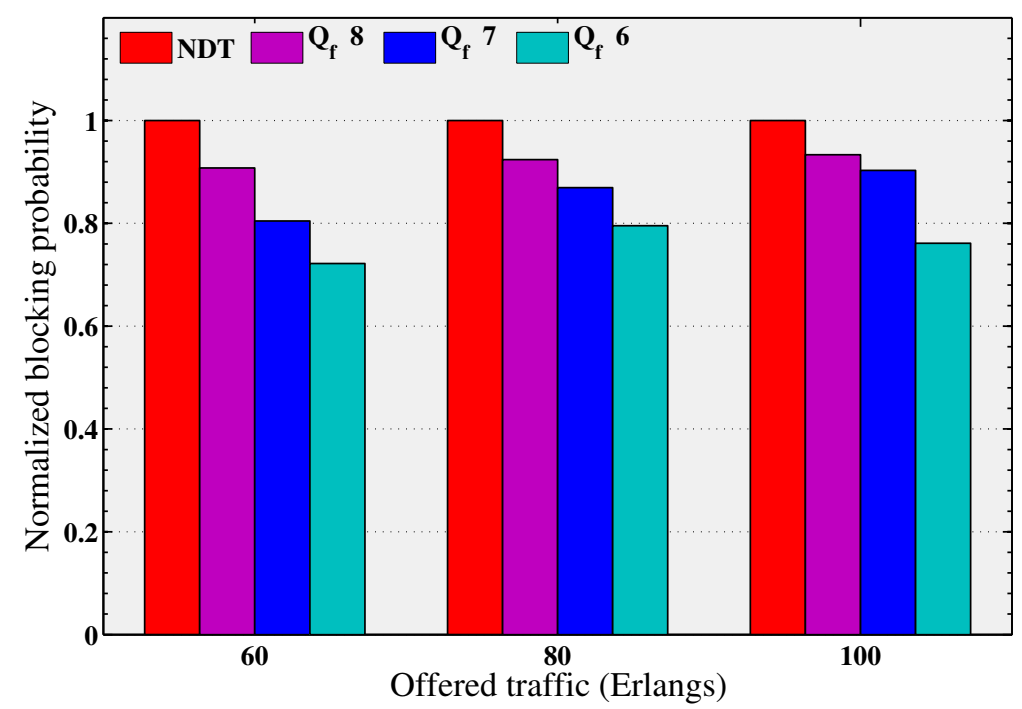

Figure 2.7: Normalized blocking probability for 14 nodes NSF topology scaled down by a factor of 10 .

fined in Paper C. Tolerable quality level $(t q l)$ specifies the slightly degraded QoT that a customer can endure momentarily in order to set-up, either its own or other connections in the network when the targeted QoT cannot be attained. Similarly, degraded quality time $(d q t)$ measures the maximum duration within the connection holding-time for which a customer can tolerate degraded QoT. These two SLA parameters can be efficiently utilized by exploiting the connection holding-time awareness.

Figure 2.7 displays the normalized BP for three different scenarios, i.e., when the receiver-end $\mathrm{Q}$-factor requirement (i.e., $Q_{f}$ ) for connection requests is equal or greater than 8,7 and 6 . The $d q t$ value for these three cases is set to $5 \%$ of the holding-time, while the QoT can be degraded down to 7.7, 6.7, and 5.7 , respectively. The BP results of the proposed QoT trading strategy are normalized to the $\mathrm{BP}$ of the basic approach, i.e., with no tolerable quality level (NTQ) connection provisioning. The results in Fig. 2.7 reveal that the network BP is significantly reduced (particularly for less stringent $Q_{f}$ case) when $t q l$ and $d q t$ are exploited for connection provisioning. An average decrease of $8 \%, 14 \%$ and $24 \%$ in BP compared to NTQ is observed for these three cases, respectively. 


\section{Chapter 3}

\section{Elastic Optical Networking}

The rapid rise in the optical networks capacity catalyzed by the WDM technology led to a wide gap between capacity and carried traffic for most of the last decade. However, the traffic growth fueled by brisk increase in the number of Internet users and emerging bandwidth intensive applications have brought networks close to the capacity limit of the conventional fiber. It is expected that the network traffic will become more diverse in terms of bandwidth granularity and temporal distribution. A near-term solution for augmenting the networks capacity requires more efficient utilization of the optical bandwidth. This ultimately translates to deriving greater flexibility from the underlying network technology. Recent innovations in optical communication systems, including advanced modulation techniques and digital equalization in the electronic domain, have facilitated per-wavelength bandwidths of 40 and 100 Gbps with improved transmission distance in WDM networks. Unfortunately, spectral efficiency higher than $2 \mathrm{~b} / \mathrm{s} / \mathrm{Hz}$ inflicts a rapid increase in the optical signal-to-noise ratio (OSNR) penalty [1]. On the other hand, further increase in the launched signal power ends in serious impairments due to nonlinear effects in the optical fibers [1]. Moreover, WDM networks exhibit a major drawback owing to their rigid and coarse granularity. Currently, wavelength-switched networks allocate a full wavelength to a connection even when the traffic between the end nodes is not sufficient to fill the entire capacity. Inverse multiplexing is employed for high rate demands, where the total rate is split into smaller chunks, such as 40 or 100 Gbps that can fit in the fixed grid. The disadvantages of inverse multiplexing are the resulting loss of spectral efficiency [45] and additional cost owing to multiple transceivers. Conclusively, wavelength level granularity leads to inefficient capacity utilization, an issue anticipated to become 
even more critical with the deployment of higher capacity WDM networks (i.e., system of 100 Gbps per wavelength and above) and higher data rate applications.

Elastic optical networks (EON) [7] have been proposed as a novel spectrum efficient and scalable optical transport solution. In the EON, the spectrum is finely divided into a number of small spectrum slices that are assigned to match as close as possible the spectrum demand of each connection. This flexibility provides several additional capabilities with respect to the fixed grid WDM network. For example, time-varying traffic can be supported by increasing or decreasing the allocated spectrum to a connection as demand alters. Furthermore, multiple data rates can be efficiently accommodated in the network, and the modulation format can be adapted in accordance to the optical reach.

Despite the fact that EON delivers many benefits, its introduction poses several challenges on the networking level and the physical layer technology. The implementation of EON necessitates innovations in network hardware and software. New components need to be developed, which might be more complex than their fixed grid counterparts. Network control and management must be evolved to fulfill the requirements of elastic networking. The rest of this chapter covers the different pieces composing the elastic networking puzzle, and reviews the various solutions suggested in the literature for these pieces. Finally, the contribution of our work in the context of dynamic bulk provisioning in elastic optical networks is also briefly discussed.

\subsection{Elastic Optical Path}

The finer granularity of network resources in EON imposes new constraints, such as spectrum contiguity and spectrum continuity for setting-up optical paths (Figure 3.1). The spectrum contiguity ensures that the assigned spectrum resources/slices to a demand are consecutive in the spectrum domain. On the other hand, the spectrum continuity inflicts that the allocated slices must be same on each link of the selected routing path. Due to the spectrum continuity constraint, there is a strong linkage between spectrum allocation and routing of a connection. Thus, routing and spectrum assignment (RSA) [46] has emerged as an important problem in EON. In the past few years, the RSA problem has been widely studied and several exact models and heuristic strategies have been proposed. RSA NP-completeness has also been proved in [47]. Furthermore, the basic RSA has been extended 


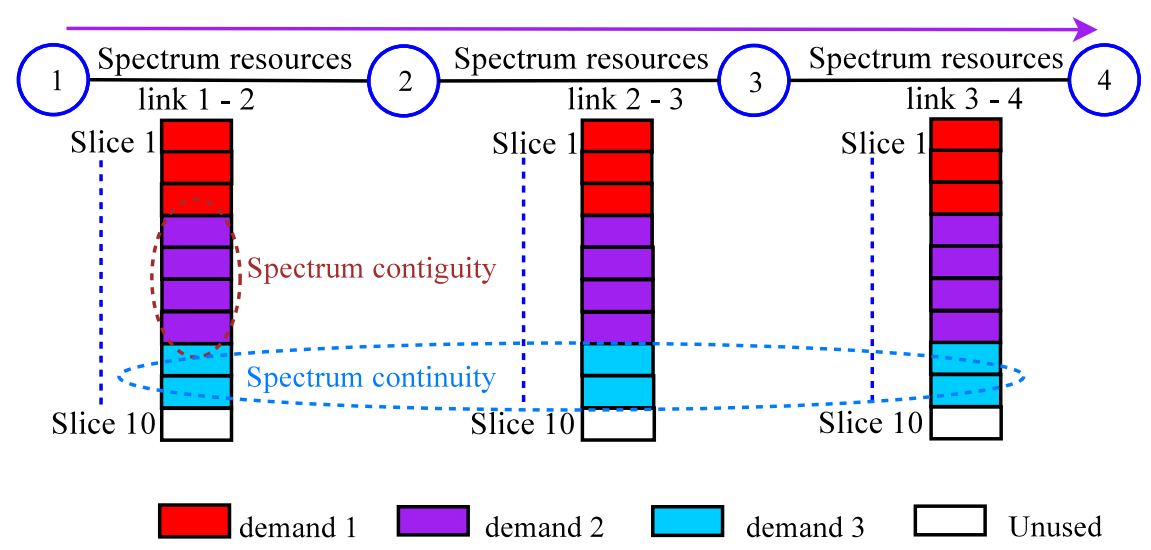

Figure 3.1: Spectrum contiguity and spectrum continuity constraints for elastic networks.

to cover additional design dimensions, such as the choice of the modulation format to be assigned to each optical path [48]. The objective of all these RSA strategies is to attain the best possible performance within the limits of the physical constraints.

Like the RWA problem, the different variants of the RSA problem can be categorized under one of the two broad versions: offline RSA, where the connection requests are known beforehand, and online RSA, in which a series of connection requests arrive in some random fashion.

\subsubsection{The Offline RSA Problem}

In the offline RSA problem, the input usually consists of a set of connection demands, and the goal is to assign a physical path and contiguous spectrum slices to each demand so as to minimize the total amount of allocated spectrum. Offline RSA problem arises when the connection demands for the network are known in advance and any variation in demand occurs over long time scale. Furthermore, these connection demands are assumed to be provisioned for relatively long periods of time, thus, it is important to optimize the allocation of network resource by developing effective solutions.

A variety of integer linear programming (ILP) formulations have been proposed for offline RSA, each tailored to a specific design aspect. Since the problem is intractable, these ILP formulations cannot be solved within a reasonable amount of time. Thus, several heuristic algorithms have been presented to achieve fairly good solutions efficiently. Like the offline RWA 
problem, the offline RSA problem is mostly solved by using the one-step approach, i.e., jointly solving the routing and spectrum assignment subproblems.

\subsubsection{The Online RSA Problem}

The online RSA problem considers a dynamic traffic scenario, where connection requests arrive stochastically into the network. Depending on the state of the network at the time of request arrival, the available spectral resources may or may not be sufficient to set-up the connection request. If a request for connection cannot be established due to lack of resources, it is blocked. The state of the network evolves randomly in time as new connections are provisioned and existing connections are released. Therefore, every time a connection request is received, an algorithm must be executed in real time to compute whether it is possible to set-up the request. Due to the real time nature of the problem, the RSA techniques must be simple and fast.

Mostly the two-step approach, i.e., tackling the routing and spectrum assignment aspects separately is employed to solve the online RSA problem. For routing the same option exist as for RWA, i.e., fixed routing, fixed alternate routing, and adaptive routing (chapter 2, section 2.1.4). Similar to wavelength assignment, there have been numerous algorithms presented for spectrum assignment. Some of them are straightforward extension of the wavelength assignment algorithms, such as First-Fit, Random, and MostUsed [20]. Other schemes specifically take into account the fragmentation issue while assigning the spectrum resources. Fragmentation-aware strategies are crucial, because scattered gaps in the spectrum can reduce the potential spectral efficiency gain of the EON concept. Examples of fragmentationaware spectrum assignment schemes are Small-Fit and Exact-Fit [49]. SmallFit allocates the smallest free spectrum block to the connection request in order to fill-up the gaps, hence, reducing fragmentation. On the other hand, Exact-Fit searches for the exact available segment in terms of the number of slices requested for the connection. If there is a segment to match the exact size of the demanded spectrum, the scheme allocates that spectrum. Otherwise, the spectrum is allocated in the first largest free block available. 


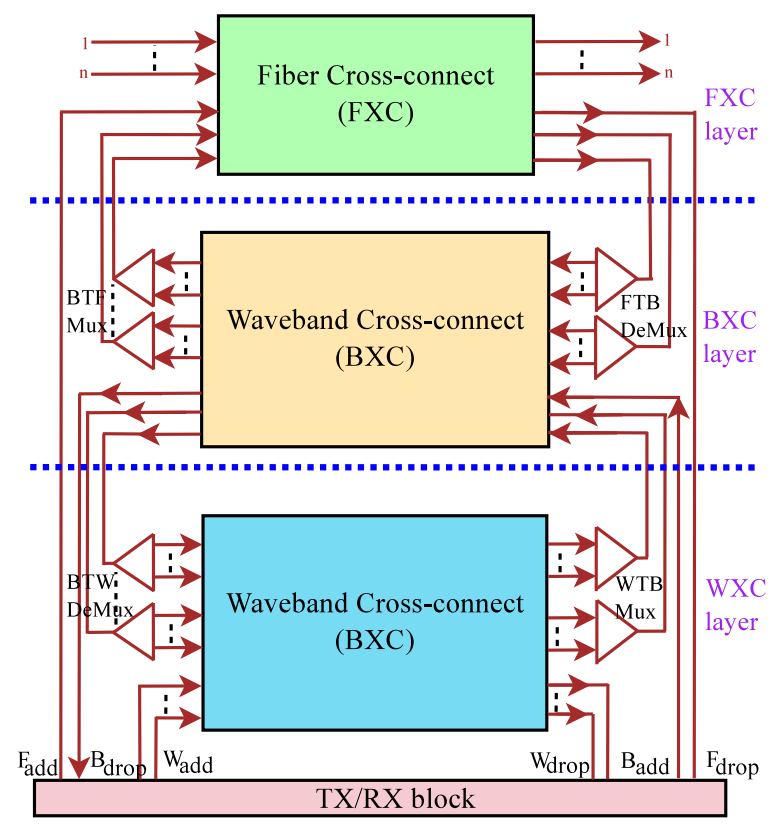

Figure 3.2: Multi-granular OXC.

\subsection{Node Architecture for Elastic Optical Trans- port}

The implementation of elastic optical transport needs the development of a new generation of optical nodes. To efficiently transport the variable size spectrum slices assigned to connections across the network, the optical nodes require active components typically based on liquid crystal on silicon (LCoS) or micro-electro-mechanical systems (MEMS). Furthermore, elastic time multiplexing necessitates fast switching devices, e.g., $10 \mathrm{~ns}$ switching time, so that to attain fine switching granularity and high efficiency. Fast time switches are generally realized with semiconductor optical amplifiers (SOA) or electro-optic materials, such as $\mathrm{LiNbO}_{3}$ or lead lanthanum zirconate titanate (PLZT).

Several approaches for the design of elastic optical nodes have been proposed in the literature. For example, evolving the traditional optical crossconnect (OXC) architectures from switching traffic all-optically at the wavelength level towards multi-granular OXC (MG-OXC) [50] that perform fiber, waveband and wavelength switching as shown in Figure 3.2. Similarly, the ROADM structure notably evolved in the past years in order to accomplish 


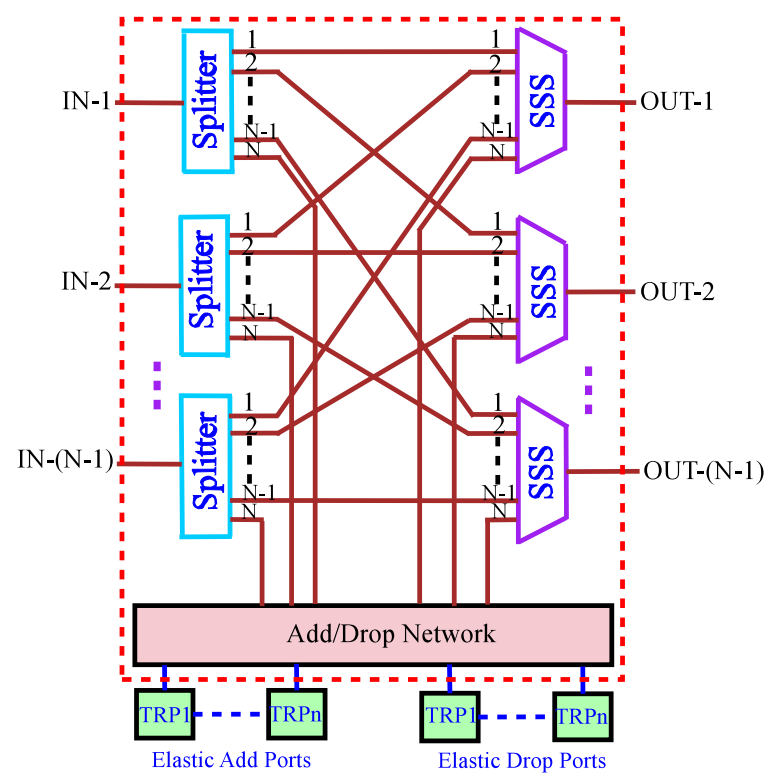

Figure 3.3: Broadcast-and-select ROADM [54].

more flexibility. Colorless, directionless, and contentionless (CDC) features are introduced to enhance the role of ROADM [51-53]. In colorless design any wavelength can be assigned to an add/drop port of the ROADM. Note that the add/drop bank provides the required connectivity between the output/input ports of the ROADM and the transmitter/receiver for connection with source/destination at the local node. A directionless add/drop arrangement provides the freedom to direct a connection to any port of the ROADM. In other words, the add/drop bank is not coupled to any specific input/output port, but shared by all the ports in the node. A contentionless ROADM design supports assignment of any wavelength to the transmitter of the add/drop bank as long as the number of connections with the same wavelength is not higher than the number of ports in the node. The proposed CDC-ROADM architectures can be upgraded to support elastic spectrum allocation by replacing the fixed-grid wavelength selective switches (WSSs) with spectrum selective switches (SSSs).

Two variants of ROADM, namely broadcast-and-select (BS) and spectrum routing (SR) are commonly considered for elastic nodes. The BS is implemented by employing optical splitters at the input ports which generate copies of the incoming signals as shown in Figure 3.3. These signals are subsequently filtered by the SSS in order to traverse appropriate signals at the output. The BS provides the benefits of being simple, however, the loss 


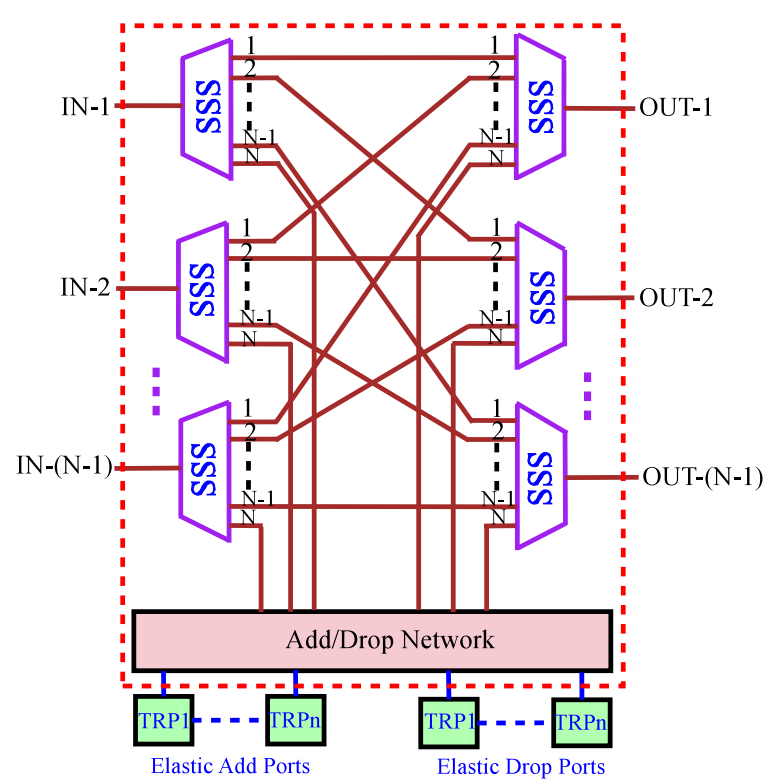

Figure 3.4: Spectrum routing ROADM [54].

inserted by the power splitters limits its scalability to a small number of nodal degrees. Moreover, as the signals are broadcasted to every output port where SSS selects specific spectrum for transmission, the port isolation of the SSS needs to be high. SR design (Figure 3.4) is a variant of the wavelength routing architecture that is realized generally with arrayed waveguide gratings (AWGs) and optical switches. However, in RS the switching and filtering functionalities are both implemented by the SSS, thus making the optical switches redundant. The fundamental advantage of SR with respect to the BS architecture is that the through loss is independent of the nodal degree. However, it needs additional SSS at the input ports, which makes the implementation more expensive compared to SR.

Another alternative proposed in the literature for the elastic optical node is the architecture on demand (AoD) [8]. The AoD architecture consists of an optical backplane (e.g., 3D-MEMS) that interconnects inputs, outputs, adds, drops, single device building modules (e.g., MUX, coupler, SSS) and composed building modules (e.g., EDFA plus splitter) as shown in Figure 3.5. With this architecture different compositions of inputs, modules, and outputs can be created by setting-up appropriate cross connections in the optical backplane. AoD provides greater flexibility than the previously mentioned architectures as the components used for optical processing, e.g., SSS, power splitters, and other functional modules are not hard-wired like in 


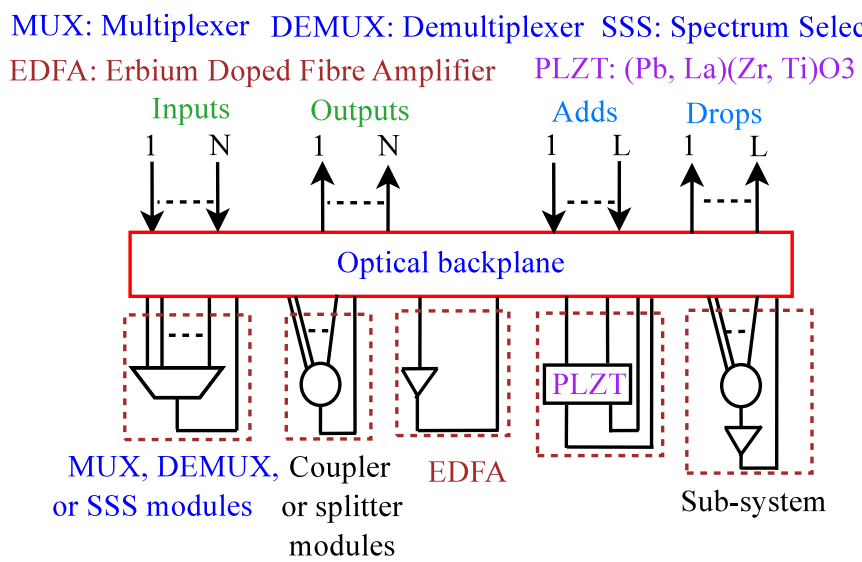

Figure 3.5: AoD implementation.

a static architecture but can be interconnected together in an arbitrary manner. In fact, it is possible to provide programmable "synthetic" architectures according to the needs of the network traffic.

\subsection{Flexible Transceivers}

Emerging digital coherent technologies exploiting digital-to-analog converters (DACs), analog-to-digital converters (ADCs), IQ-modulators, and digital signal processing (DSP) have led to a new degree of freedom for designing optical transceivers in the electronic domain. However, due to technological limitations, such as the maximum sampling rate of (ADCs/DACs), the concept of superchannels has emerged for higher data rate channels. Superchannels are essentially a combined set of tightly spaced subchannels which are commonly known as optical subcarriers. Superchannels are extremely important to cater demands beyond $100 \mathrm{Gbps}$. The symbol rate, modulation format (i.e., bits per symbol), and number of subcarriers can be adjusted to achieve desired optical reach and/or adaptive data rate optical transceivers. There are two options for elastic optical transceiver design: constant spectral occupancy/adaptive capacity and constant capacity/adaptive spectral occupancy. In the former case, the symbol rate is fixed and the data rate is adapted by changing the modulation format while ensuring the required optical reach. In the latter case, the symbol rate and the modulation format are adjusted to minimize spectral occupancy while guaranteeing the essential optical reach. 


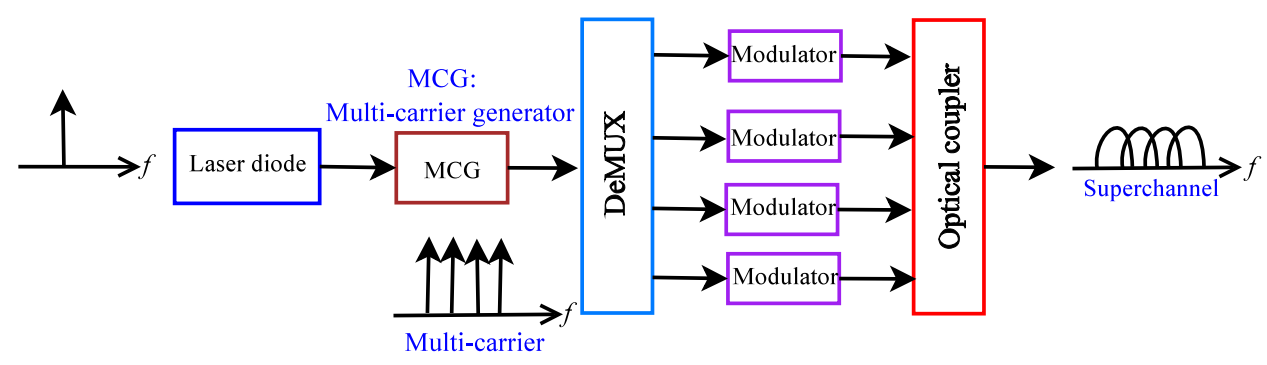

Figure 3.6: OFDM-based transmitter architecture [55].

There are two schemes for achieving an optical bandwidth variable transceiver (BVT), namely, optical OFDM (O-OFDM) and Nyquist WDM (N-WDM) [55, 56]. In O-OFDM, the optical signal is transported on a number of low rate orthogonal carriers, the bandwidth of which can be increased or decreased by adding or deleting carriers (Figure 3.6). The orthogonality of the carriers allow them to partially overlap in the frequency domain, hence providing a spectrally efficient means of traversing a high data rate signal. In addition, the requirement for the speed of the underlying electronics can be lower owing to the lower rate of the constituent carriers, making it less technically challenging to acquire very high bit rate. However, a frequency locked multi-carrier generator is needed to create carriers that satisfy the orthogonal condition. N-WDM (Figure 3.7) utilizes carriers which are conventional WDM channels, spectrally shaped to produce close to a rectangular shape (in the frequency domain) so that to allow the carriers to be tightly packed. The bandwidth of each carrier must be close to Nyquist limit in order to avert inter-symbol interference, which coincides with the baud rate. The carriers do not need to be frequency locked, thus, individual laser diodes can be employed for each carrier. Sharp roll-off optical filtering, electrical Nyquist filtering, or electrical OFDM can be exploited to generate the bandwidth limited almost rectangular spectrum pulse.

Several demonstrations of variable bit rate transmitters have been reported, where the number of carriers or the modulation format is adapted to attain the desired bit rate and spectral efficiency $[56,57]$. However, operation of high speed BVT below its maximum rate due to targeted reach or impairments in the optical path leads to partly waste of BVT capacity. To address this problem, a multi-flow transponder or sliceable BVT (SBVT) [55] has been proposed. A SBVT can assign its capacity to one or multiple optical connections (flows), which are then carried to one or several destinations. This enables SBVT to employ the remaining capacity for transmitting other 


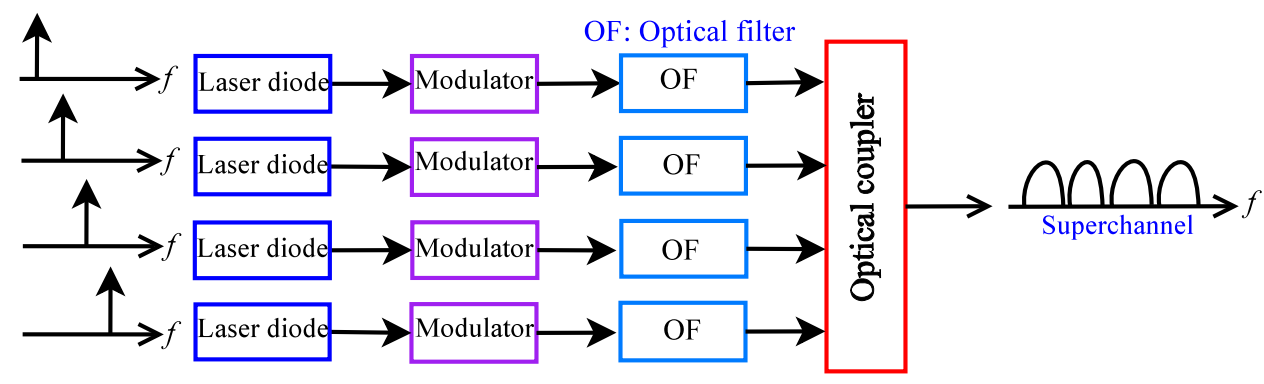

Figure 3.7: Nyquist-WDM-based transmitter architecture using optical filters [55].

independent flows, when it is used for generation of low bit rate connections. Depending on the mode of operation, a SBVT can be viewed either as a high capacity BVT or as a collection of multiple virtual independent lower capacity BVTs. Moreover, the implementation of SBVT is achievable through commercially available technologies, such as photonic integrated circuits (PIC). Preliminary studies indicate that the SBVT model is more cost-efficient compared to other alternatives by virtue of its capability to handle multiple optical paths and better resource sharing [58].

\subsection{Control Plane for Elastic Networking}

The control plane solutions for WDM networks cannot be precisely applicable to EON due to its specific characteristics. Research efforts for EON control plane architecture have been focusing on extension of the current available architectures, i.e., GMPLS along with novel solutions, such as software-defined networks (SDN) [59]. The GMPLS protocol suite is the most adopted control plane for optical networks owing to its vast functionality, and is considered as a natural choice for implementation of EON control plane. However, GMPLS for EON must consider spectrum slices rather than wavelength, which may influence the scalability issues. The control plane protocols will need to maintain coherent global information about up to 320 or 640 slices in the $12.5 \mathrm{GHz}$ or the $6.25 \mathrm{GHz}$ slice granularity, respectively [60]. In addition, precise tracking of the spectral width and center frequency for optical signals will be required. For this purpose, a considerable effort is being conducted in IETF to extend GMPLS to incorporate the flexible optical switching capabilities. A new dense WDM grid has been developed within the ITU-T Study Group 15 by defining a set of nominal 


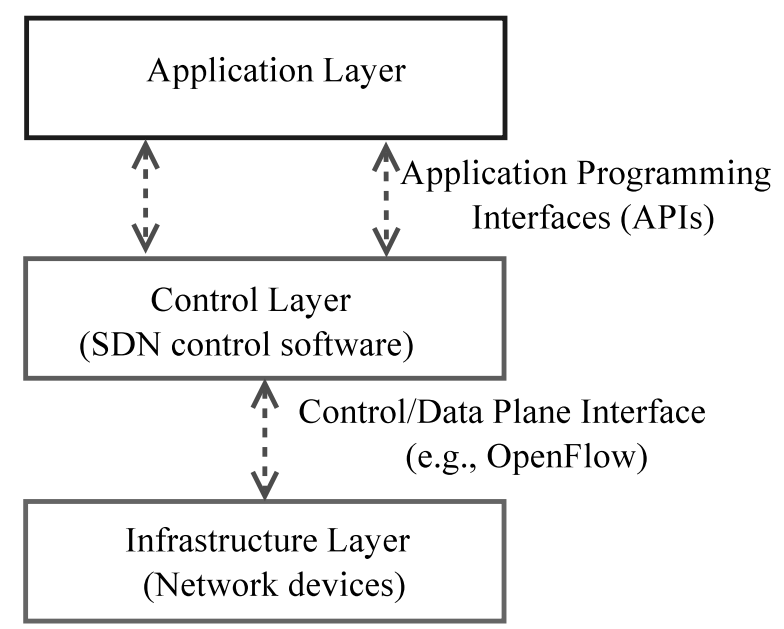

Figure 3.8: Three-layer SDN architectural model [3].

central frequencies, channel spacing, and the concept of spectrum slice [61]. Moreover, extensions to the PCE communication protocol (PCEP) have been present in [62] to enable dynamic spectrum assignment and modulation format adoption.

A new paradigm for control plane architecture is the SDN, which decouple the network control plane and data plane while employing the centralized concept for network control. In the SDN model, the network devices would be controlled through software that operates in a decoupled control plane. The SDN control software (i.e., the SDN controller) is basically a network wide operating system that executes tasks, such as topology discovery, routing, traffic engineering, and recovery. In addition, it is capable of populating the forwarding tables of the network devices, enabling them to perform very fine granularity control of the network flow. The concept of SDN can be comprehended as a three layered architectural stack, as shown in Figure 3.8. The network applications are at the top, while the infrastructure layer composing of network devices is at the bottom. The SDN control layer is in-between, responsible for presenting a vender independent interface, a network wide view to the applications, and translating requirements of the applications into instructions for the infrastructure layer. The most popular protocol for the control plane/data plane interface is OpenFlow. OpenFlow provides direct access to the data plane of the various networks elements, thereby allowing the software controllers to program these elements. This facilitates the software to adjust traffic flow based on factors, such as usage pattern, application, required resources, or business policy. 
There are numerous potential benefits that can be achieved by decoupling the control and data planes. For example, it allows the control software to be more easily modified or customized, thus, providing greater control to carriers of their network to introduce new services and innovations. Network management will be simpler and more automated with less chance for manual configuration errors. In addition, SDN is an enabler of dynamic networking. Finally, taking the control functionality out of the network devices might reduce their cost.

\subsection{Spectral Defragmentation}

During network operation, connections with different spectrum requirements are set-up and torn down; breaking the spectrum into small sized blocks of free slices which might be misaligned with the available slices on the adjacent links. Spectrum fragmentation aggravates the blocking performance; the links may have free slices, but the lack of contiguity/continuity in the spectrum may lead to dropping connection requests (Figure 3.9). Various strategies have been proposed in the literature to address the fragmentation issue. The fragmentation-aware routing and/or spectrum assignment techniques proposed in $[49,63,64]$ aim at mitigating the spectrum fragmentation to lower the number of blocked connection requests. Similarly, spectral defragmentation approaches [65-67] reroute and/or reallocate spectrum to existing connections in the network, whenever the connection request blocking level in the network exceed a certain threshold or a new demand request cannot be accommodated. The former approach is considered as active, while the latter is termed as reactive.

Different methods for quantifying the level of fragmentation in EON have been presented. For example, network spectrum compactness [68] and utilization entropy [69] which measure how well the spectrum resources are occupied, can be employed as parameters for triggering the defragmentation algorithms. In addition, these metrics would be used to assess the performance of the fragmentation-aware routing and spectrum assignment techniques. In this context, Paper $\mathbf{D}$ and $\mathbf{H}$ employ network utilization entropy for evaluating how good the proposed strategies are in terms of resource fragmentation. Network utilization entropy is defined as the sum of variations in the usage status of all pairs of neighboring slices on every link divided by the product between the total number of fiber links in the network and the number of available slices per link reduced by 1 . A lower 


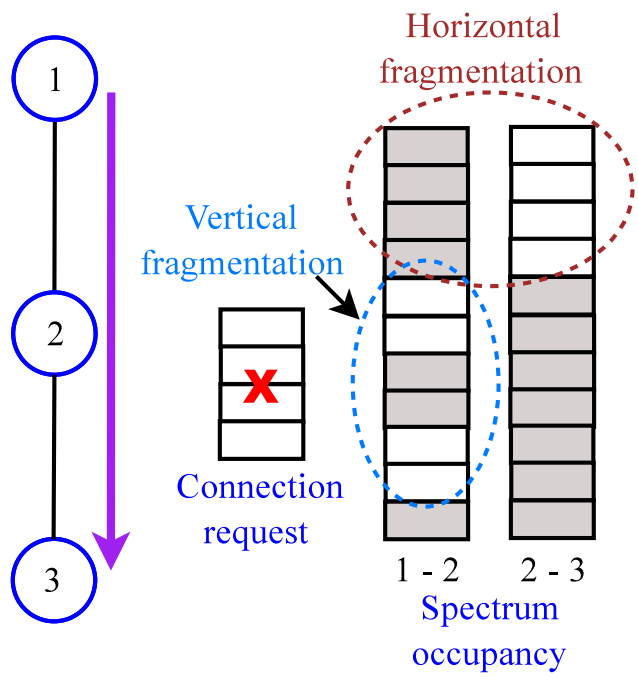

Figure 3.9: Fragmentation problem in elastic networks.

utilization entropy indicates that the resources are used in a more ordered form, with fewer gaps in the slice map and a lower level of fragmentation.

To minimize the connection request blocking and the fragmentation level in the network, Paper D investigates the benefits of set-up delay tolerance in EONs allowing for dynamic bulk provisioning of connection requests. The elastic bulk provisioning (EBP) problem is formalized as an ILP, and the set-up delay tolerance is employed as a time threshold parameter for collecting and creating bulks of connection requests. Connection requests arriving in the network are accumulated for concurrent optimization until the set-up delay tolerance of the most impatient request expires. The bulk of requests is then fed to the ILP solver for routing and spectrum assignment. The objective function of the ILP formulation is set to minimize the total bandwidth of all blocked requests and to lower the spectrum fragmentation by employing the Exact Fit [49] spectrum allocation policy. The impact of spectrum selection policy in the objective function is incorporated by employing a tunable parameter $\gamma$.

The performance of the EBP approach is assessed in terms of bandwidth blocking ratio (BBR), defined as the ratio between total blocked and requested bandwidth, and of network utilization entropy. Simulation results are compared against a baseline (BL) approach that employs the same objective function, but provisions connection requests one by one as they arrive in the network. The value of set-up delay tolerance $\left(t_{d}\right)$ for each request is set to $5 \%$ or $10 \%$ of the connection holding-time $\left(t_{h}\right)$. 


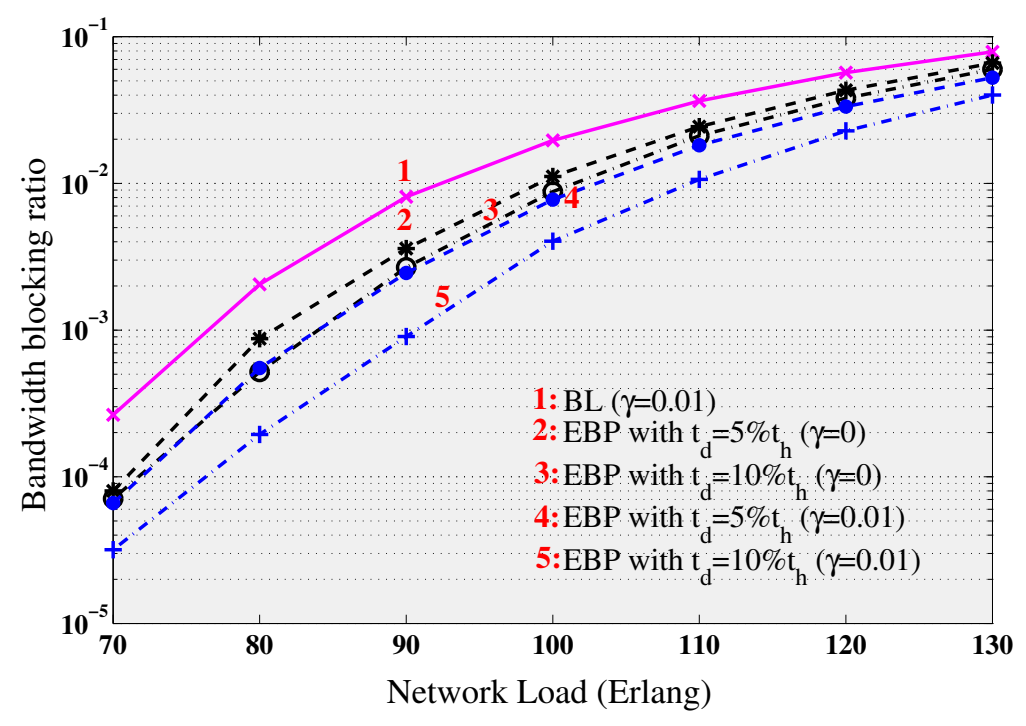

Figure 3.10: Bandwidth blocking ratio vs. offered network load for 14 nodes NSF topology.

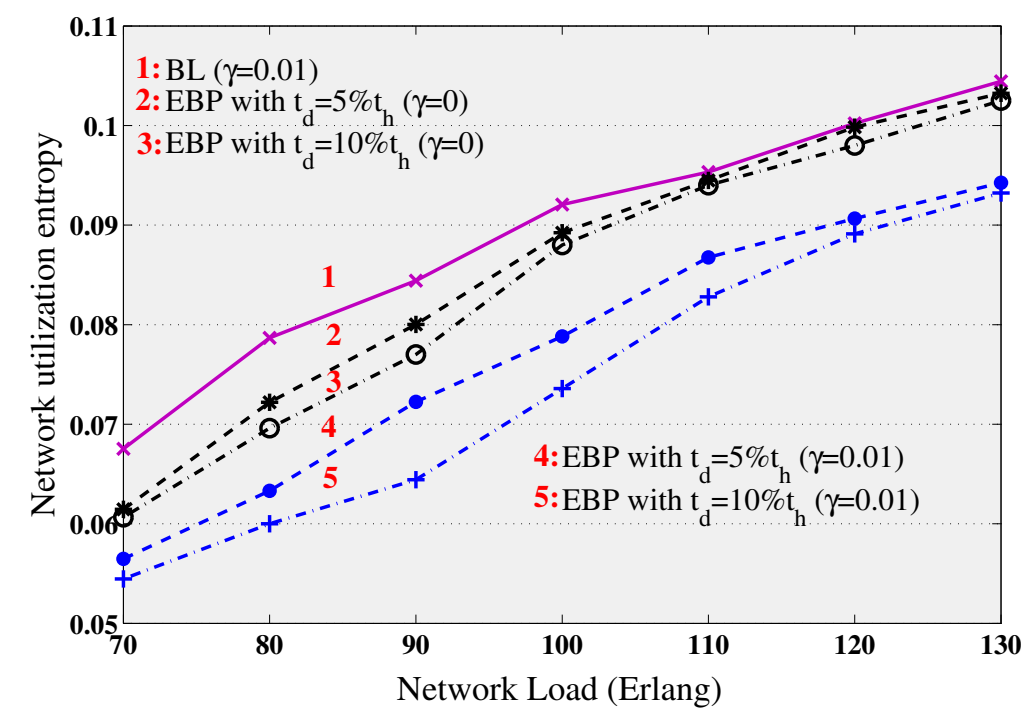

Figure 3.11: Network utilization entropy vs. offered network load for 14 nodes NSF topology. 
The average value of BBR as a function of the load for 14 nodes NSF topology is evaluated in Figure 3.10. The figure shows that allowing larger values of the time-threshold parameter (i.e., $10 \%$ of $t_{h}$ ) can augment the size of the bundle of connection requests for concurrent optimization, and consequently enhance the network BBR performance. Moreover, concurrent optimization of connection requests considering only resource utilization (i.e., for $\gamma=0$ ) still has the potential to improve BBR compared to sequential provisioning (i.e., BL) which considers both resource and fragmentation minimization. Finally, bulk provisioning which additionally minimizes fragmentation (i.e., for $\gamma=0.01$ ), can bring further and significant reductions of the BBR. This indicates that maintaining a low level of fragmentation decreases the chance of rejection for incoming/future connection requests, which in turn leads to an overall improvement in BBR. This latter aspect is further verified by the network utilization entropy values shown in Figure 3.11, with the EBP with $\gamma=0.01$ outperforming all other approaches. 


\title{
Chapter 4
}

\section{Architecture on Demand - Network Features}

\begin{abstract}
Architecture on Demand (AoD) is a promising solution for future high capacity flexible and evolvable networks. The node modules of AoD are not hard-wired, but can be connected/disconnected to any input/output port according to the requirements. Thus, each AoD node and the network (fabricated with AoD nodes) as a whole acts like an optical field-programmable gate array. This flexibility inherent by AoD offers several advantages over static node architectures, such as enhanced scalability, cost effectiveness, energy-efficiency, and better resiliency. Paper E and Paper F of this thesis work investigate some of these aspects for AoD based networks in the context of network planning and operation.
\end{abstract}

\subsection{Scalability Analysis of AoD Based Networks}

The versatile feature of AoD enables the node to switch the optical signals in a given input port without utilizing any demultiplexing and multiplexing device if all these signals traverse through to the same output port with no signals from other input ports. Such an operation called fiber switching, employs only a cross-connection in the optical backplane for connectivity between the input/output ports (Figure 4.1). This aspect of AoD can improve the node and consequently the network scalability. In addition, the number of hardware modules for the network can be reduced significantly, thus, making it cost-effective. 


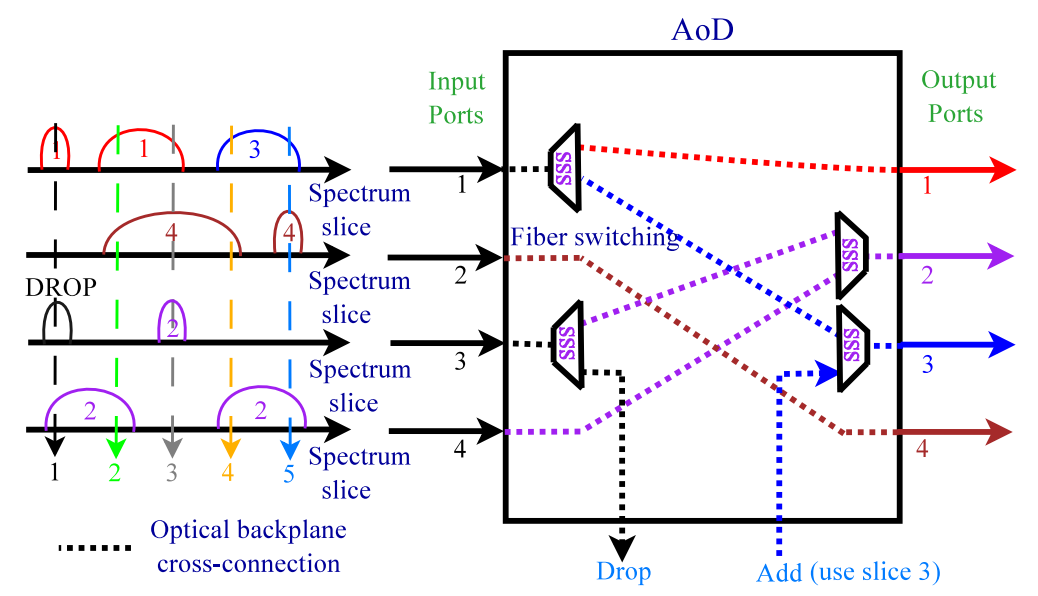

Figure 4.1: Example of AoD configuration for a specific set of connections.

The first part of Paper E presents a cost-efficient design strategy for evolvable networks with AoD nodes, which composes and dimensions the network by allocating component functions on a per-node basis. The network planning problem is defined through an ILP formulation and is solved in a real case scenario. To encompass the scalability limitation by the optimal solution based on the ILP formulation, a scalable and efficient heuristic algorithm is also proposed. The heuristic algorithm chooses the route and assign spectrum to connection requests with the aim to maximize the fiber switching operation, and is able to well approximate the optimal solution. Figure 4.2 shows the average module savings for different values of network utilization for the heuristic strategy. Note that for the comparison of the performance of the proposed heuristic strategy for different topologies, the network utilization instead of number of connection requests is employed. The network utilization is computed by measuring the percentage of the number of spectrum slices occupied by the established connections out of the total number of slices available on all the links of the network. Furthermore, for benchmark purpose, AoD architecture with switching modules at each input/output port is considered. At low loads, the heuristic solution saves up to $70 \%$ of modules with respect to the benchmark for both of the network topologies. However, for higher loads the gain drops to $40 \%$ and $25 \%$ for COST239 and NSF, respectively. However, this signifies a major cost saving especially for scalable and evolvable networks. This also indicates that the proposed strategy is more effective in terms of reducing the network modules for topology with high nodal degree (i.e., COST239). In fact for highly connected networks the solution space is larger, which facili- 


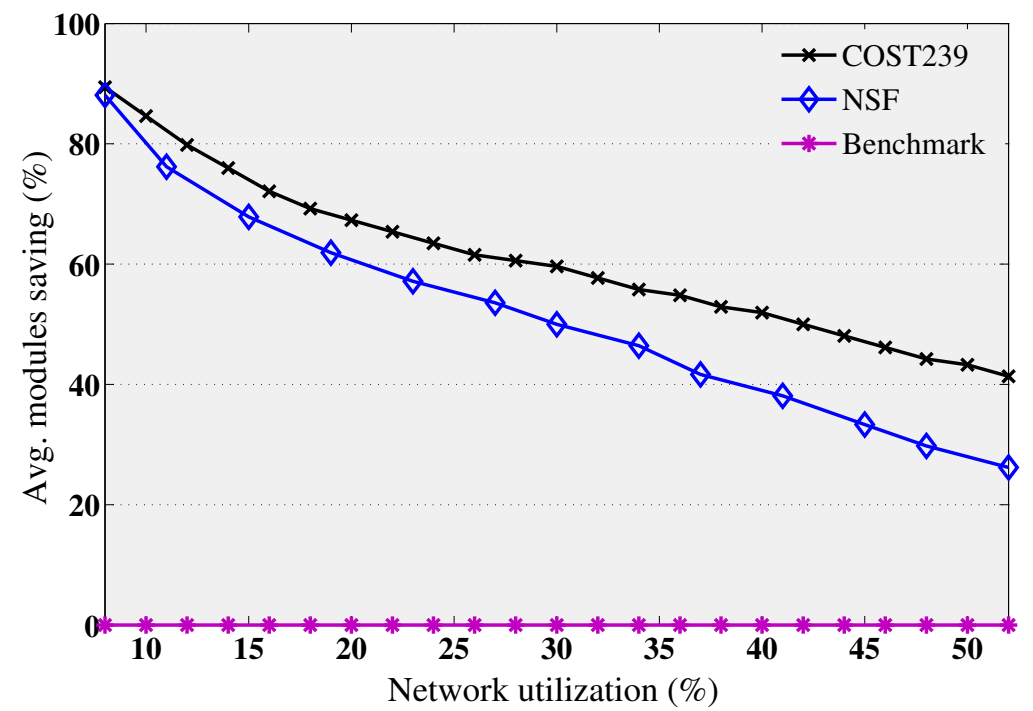

Figure 4.2: Average switching module savings versus network load, for NSF and COST239 topologies.

tates the strategy to select the best paths for demands in terms of switching modules.

Figure 4.3 and Figure 4.4 evaluate the number of cross-connections per node required to implement AoD for NSF and COST239 topologies, respectively. Notice that the number of cross-connections indicates the optical backplane port count, and thus can be considered as a scalability parameter for AoD. The figures depict that the heuristic strategy has the capability of reducing the number of cross-connections enormously compared to the benchmark. Even for a high nodal degree node (i.e., $\mathrm{N}=6$ ) the strategy managed to scale down the number of cross-connections by half, through utilizing the versatility offered by AoD. Furthermore, the number of required cross-connections (shown in the figures) indicates that the networks designed and studied are realizable with commercially available optical backplanes.

\subsection{Power Consumption Analysis of AoD Based Networks}

Taking advantage of the agility embodied in AoD through the proper RSA technique, the active switching modules are deployed where and when needed 


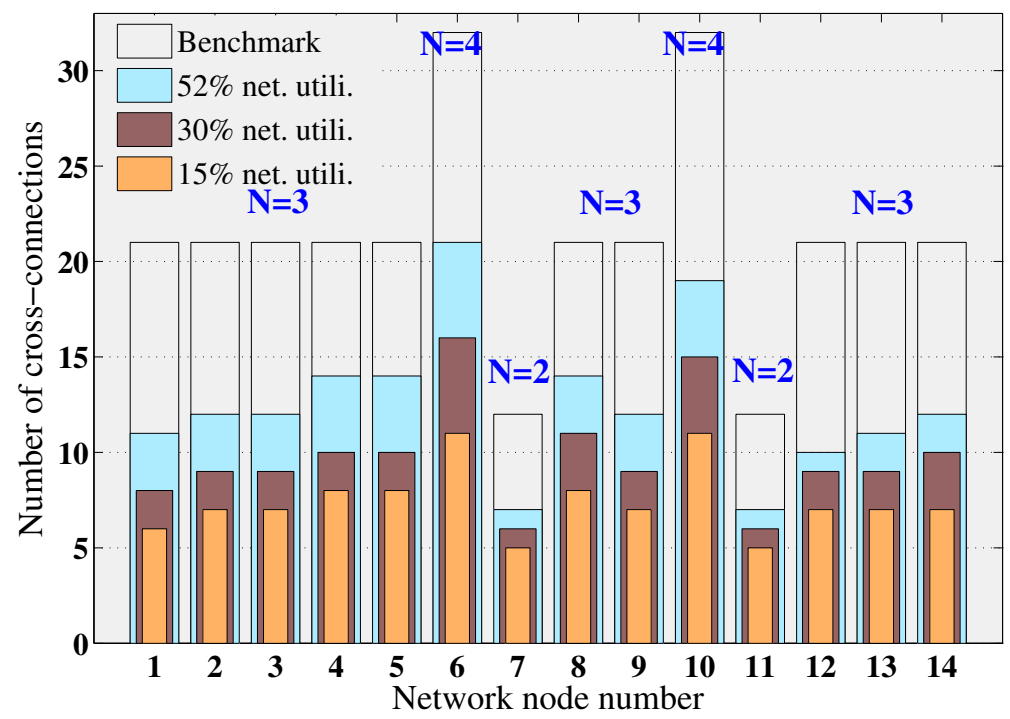

Figure 4.3: NSF topology: numbers of cross-connections per node versus network node number.

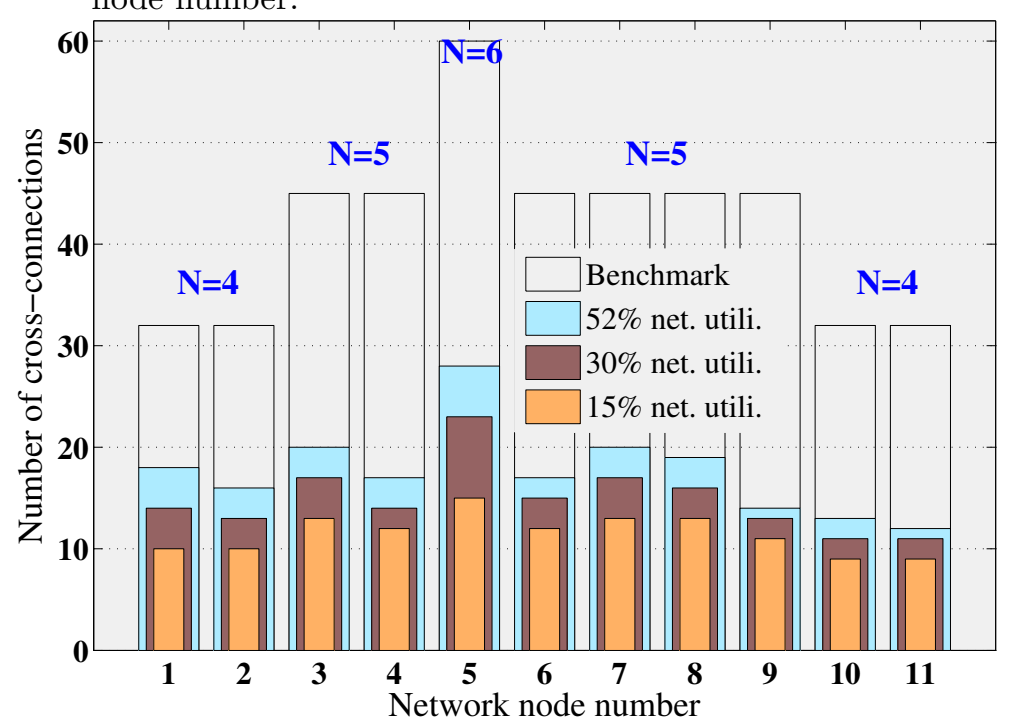

Figure 4.4: COST239 topology: numbers of cross-connections per node versus network node number. 


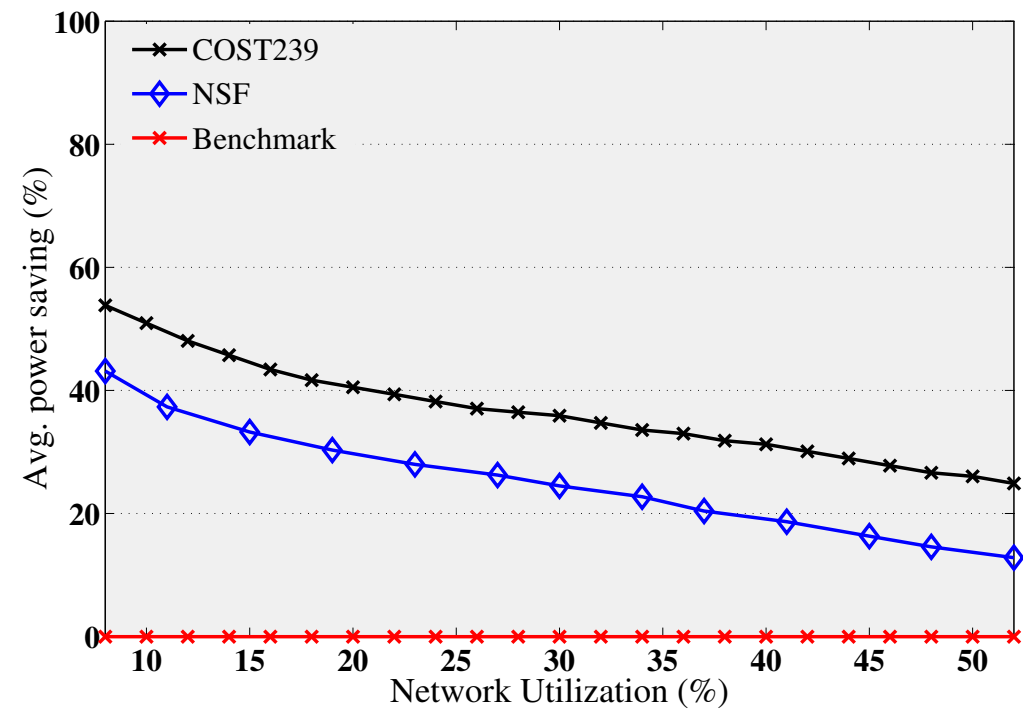

Figure 4.5: Average power saving versus network load, for NSF and COST239 topologies.

Table 4.1: Power Consumption Contributions

\begin{tabular}{|c|l|}
\hline Device & Power [W] \\
\hline Common equipment & 100 \\
SSS [70] & 40 \\
3D-MEMS(320 page) [71] & 150 \\
\hline
\end{tabular}

in the network. Thus, unlike the static nodes network, the power consumption of the network fabricated with AoD nodes depends on the traffic demands. Figure 4.5 shows the average power saving as a function of network utilization for NSF and COST239 topologies. The parameters characterizing the power consumption are set according to the values highlighted in Table 4.1. Notice that for the power saving only the devices installed in the nodes are considered without taking into account the link devices (e.g., optical amplifiers). Figure 4.5 reveals that at low loads, the network with fabricated AoD nodes saves up to $50 \%$ and $40 \%$ for the COST239 and NSF topologies, respectively. These values drop to $30 \%$ and $20 \%$, respectively, for higher loads. 


\subsection{Performance for Dynamic Scenario}

Unlike the planning case in which the demands are known beforehand, for the dynamic traffic scenario connection requests arrive and depart from the network stochastically. Thus, when applying cost-efficient techniques (i.e., maximizing fiber switching operation) in dynamic networks, the drawback is a possible degradation of the network performance in terms of blocking probability. In fact, in dynamic networks with AoD nodes the blocking of requests can be caused by two factors: i) an insufficient number of switching modules at AoD nodes to support the required connectivity; and ii) a shortage of spectrum resources along the route between the source and destination node of a connection. The blocking caused by the former case can be prevented by augmenting the switching modules in the network nodes, and through an efficient routing algorithm that is tailored to make the best of the deployed switching module.

The second part of Paper E presents a two-step approach for improving the blocking probability caused by the scarcity of switching modules in AoD nodes in a dynamic environment. The first step is to efficiently design the network nodes for a specific offered traffic. This is done by executing the heuristic planning strategy for numerous sets of random connection requests (static traffic) of the same intensity as that of the offered load. Such an approach facilitates estimating the best location for deployment of switching modules; i.e., the nodes are synthesized from the modules that attain high frequency for the sets of random demands. The switching devices once assigned to the specific input/output ports (of a given node) will not be moved to other ports; however, their interconnectivity can be altered. Once the network is designed, then the next step is to apply the proposed provisioning strategy, i.e., shortest path with minimum fiber switch (SPFS), which is specifically tailored to facilitate the usage of the installed switching modules in the nodes for dynamic traffic. Note that SPFS uses the designed network (step 1) as an input and has no further relation with the first step. The SPFS algorithm aims at finding for each arriving request a path able to satisfy the spectrum contiguity and continuity constraints while keeping both the number of used resources and the fiber switch at a minimum.

The performance of the proposed strategy is assessed in terms of the probability of rejecting requests due to an insufficient number of switching modules at AoD nodes. Furthermore, for other alternatives, such as the broadcast and select configuration for CDC-ROADM and the AoD with switching devices at each input/output port, the blocking of demands due to the switching 


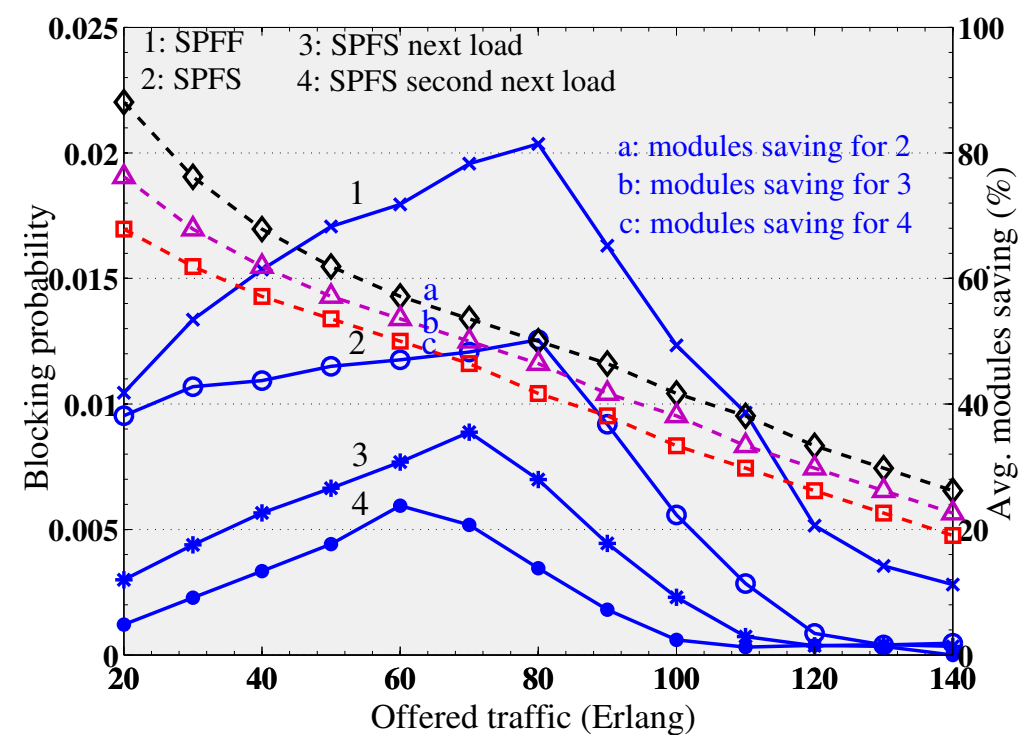

Figure 4.6: NSF topology: blocking probability (LHS) and average module saving (RHS) versus offered network load.

module constraint is equal to zero. Therefore, for benchmarking purposes SPFS is compared with the basic approach, i.e., shortest path with first fit (SPFF), for the same fabricated network. The SPFF strategy employs the First-Fit spectrum assignment technique [27] for allocating spectrum available on the very first shortest feasible path for demands. Figure 4.6 displays the network blocking probability for the NSF topology when the network designed for static load is used for dynamic traffic of the same intensity. In other words, for 20 Erlang load, the offline technique is operated for random sets of 20 connections (static traffic), and the same method is adopted for other load cases. The same figure also shows the module savings (y axis on the right) compared to AoD nodes with no fiber switch operation, i.e., nodes designed with theoretical allocation of modules. It can be seen from the figure that the proposed strategy, SPSF, exhibits better performance compared to SPFF. Furthermore, the blocking probability curve first slowly rises, and then beyond 80 Erlang it sharply declines. This anomaly is due to the fact that at low loads the number of switching modules deployed for the static case is relatively small (Figure 4.2), yielding more restricted intra-nodal connectivity and high device savings (curve a). In turn, the connectivity is not good enough to handle the routing for the stochastic arrival and departure of demands into the network. However, as the number of switching components increases with load the savings in devices decreases 
but the intra-nodal connectivity and consequently the blocking performance of the network improve.

To analyze the trade-off between the blocking performance and switching module savings curves 3 and 4 (Figure 4.6) show the blocking probability of the network designed with supplementary switching devices. Curve 3 manifests the blocking probability when the network planned for 30 demands (static traffic) is operated for 20 Erlang traffic, and the same difference is also maintained for the other offered traffic cases. Similarly, curve 4 demonstrates the blocking performance when the network designed for 40 demands is utilized for 20 Erlang traffic, and the same inequality is also kept for the other offered traffic cases. It is obvious from the figure that the blocking performance of the network drastically improves with a slight decrease in the network module savings (curves b and c). For high loads, the blocking of demands due to the switching modules constraint approaches zero; still the savings is more than $20 \%$.

\subsection{Survivability Analysis of AoD Based Networks}

AoD offers new trends for improving network reliability by supporting nodelevel recovery, i.e., self-healing from failures of node components. Self-healing is based on the possibility to switch over the connections afflicted by failures, to the node redundancy that is either deployed in the node deliberately for survivability purposes or generated by releasing the unused components. The optical backplane can easily be reconfigured to employ the redundant component, enabling fast failure recovery in $10 \mathrm{~ms}$ without activating network wide survivability techniques [8]. Furthermore, avoiding the usage of unnecessary node components (whose failures affect a connection) by promoting fiber switching increases the network availability. In other words, enhancing fiber switching improves network availability, as a decrease in the number of components traversed by a connection reduces the related risk of failure. However, as explained previously, replacing modules with fiber switching might lead to a degradation of the network blocking performance in dynamic network conditions, where connection requests arrive and depart stochastically. Blocking inflicted by insufficient number of switching modules at AoD nodes can be prevented by increasing the number of switching modules in an AoD node. Nevertheless, due to the flexibility of AoD nodes, another option is to utilize modules dedicated for protection to serve connection requests that would otherwise be blocked due to the restricted port connectivity. 


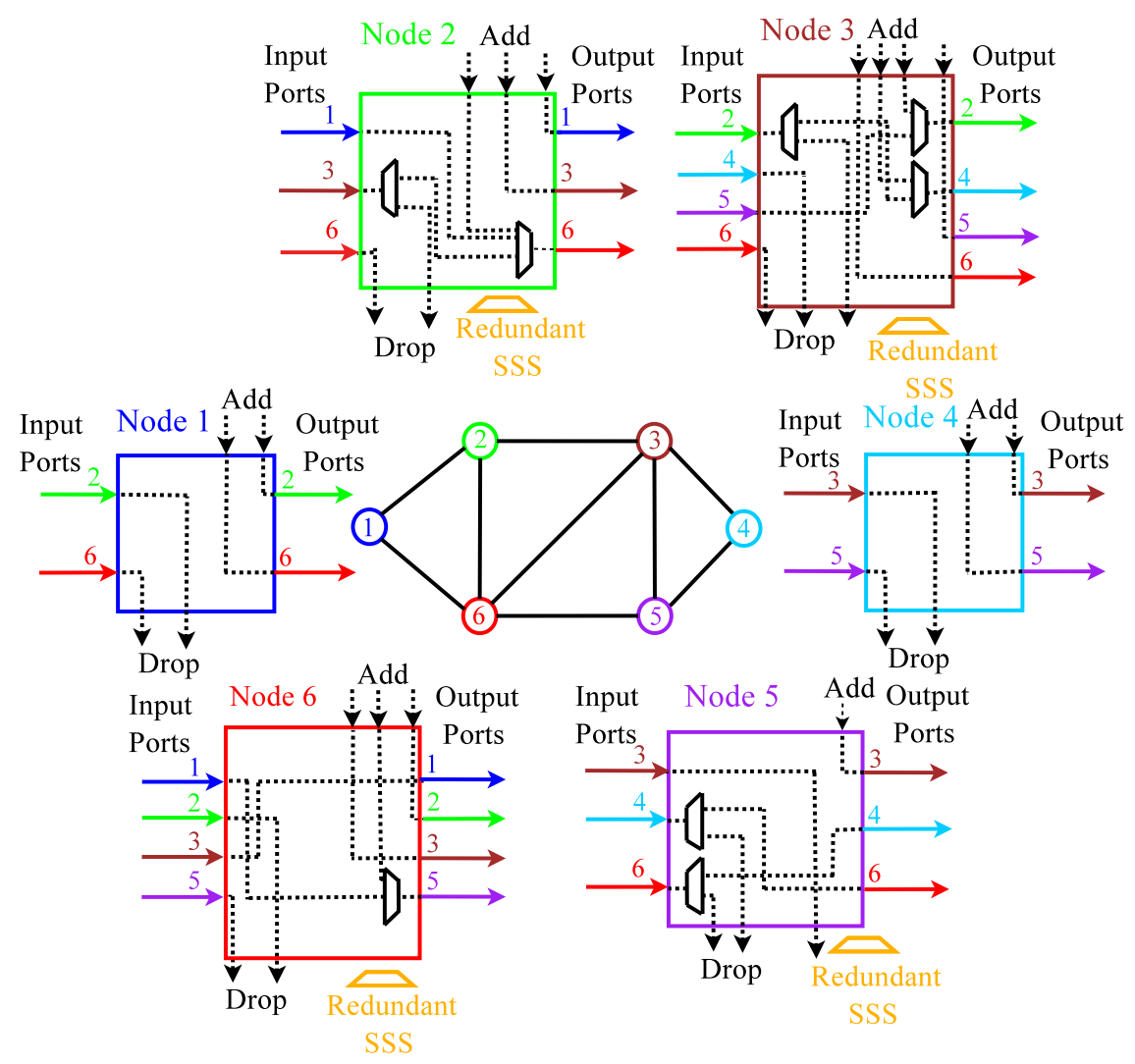

Figure 4.7: Sample network with AoD nodes.

Figure 4.7 depicts a sample network with AoD nodes that are configured to fulfill the provisioning requirements of the established connections. If a new request from source 1 to destination 3 arrives into the network, then for the given network configuration there is no viable path from node 1 to node 3 . However, the redundant devices at nodes 5 and 3 are idle. Taking the advantage of this situation, the proposed strategy reconfigures nodes 5 and 3 (Figure 4.8) by coupling the redundant devices with their output port 3 and input port 5 , respectively. Thus, making it possible to set-up a new connection from source 1 to destination 3 , and improving the network BP. However, such utilization of redundant components might render them unavailable for accepting traffic from failed working components within the node, which can in turn decrease connection availability.

Paper F presents a dynamic connection provisioning strategy (i.e., dynamic provisioning with preemptable spectrum selective switches, or DP-PSSS) 

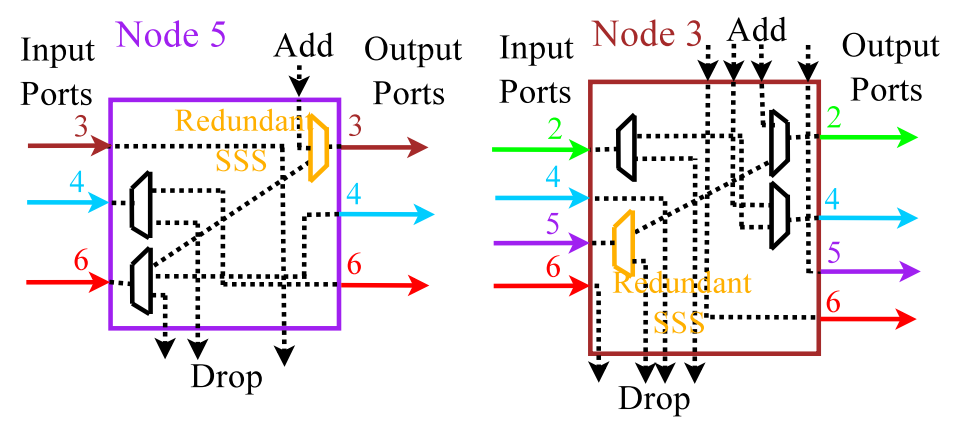

Figure 4.8: New configuration for node 5 and node 3 .

aimed at improving blocking probability caused by the scarcity of switching modules in AoD nodes, while balancing the trade-off with connection availability. The strategy utilizes redundant switching modules deployed in a survivable AoD nodes to accommodate the requests which would otherwise be blocked. By preempting the connections established by a redundant module in order to protect connections served by a failed working module, the proposed strategy is capable of obtaining an advantageous trade-off between blocking probability and network availability.

Figure 4.9 shows the average network blocking probability for the NSF topology for two scenarios, i.e., when AoD nodes can have at most (i) one or (ii) two redundant SSSs. For each load value the dynamic provisioning of connections is simulated with the number and placement of SSSs determined in the design phase. DP-PSSS achieves a significant reduction in blocking probability, i.e., an average decrease of $53 \%$ and $62 \%$ with one and two redundant modules per node, respectively. Figure 4.10 exhibits the average connection availability (i.e., the ratio between the time a connection is operative over the entire connection holding time) as a function of the load. The benchmark strategy, which employs the redundant resources only for failure recovery displays $100 \%$ availability. The lower blocking obtained by DP-PSSS comes at the expense of slightly degraded availability due to preemption of connections which traverse a backup SSS in the event of a working module failure. However, this degradation is relatively contained, as it is shown in Figure 4.10 that the average value of the connection availability never drops below 99.99. 


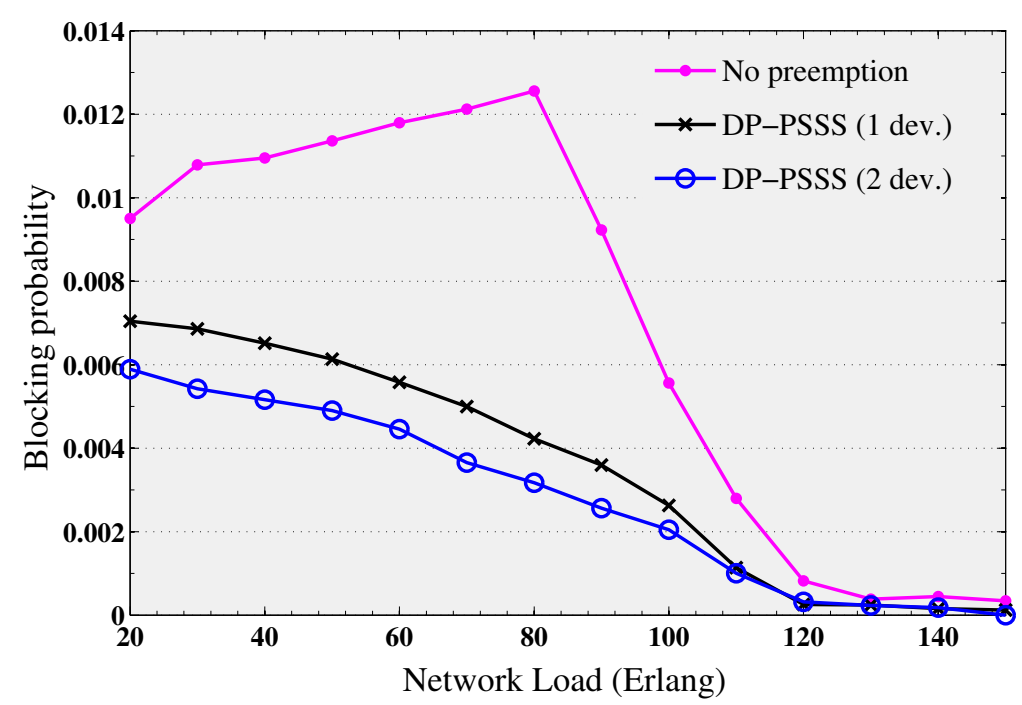

Figure 4.9: Blocking probability vs. offered network load.

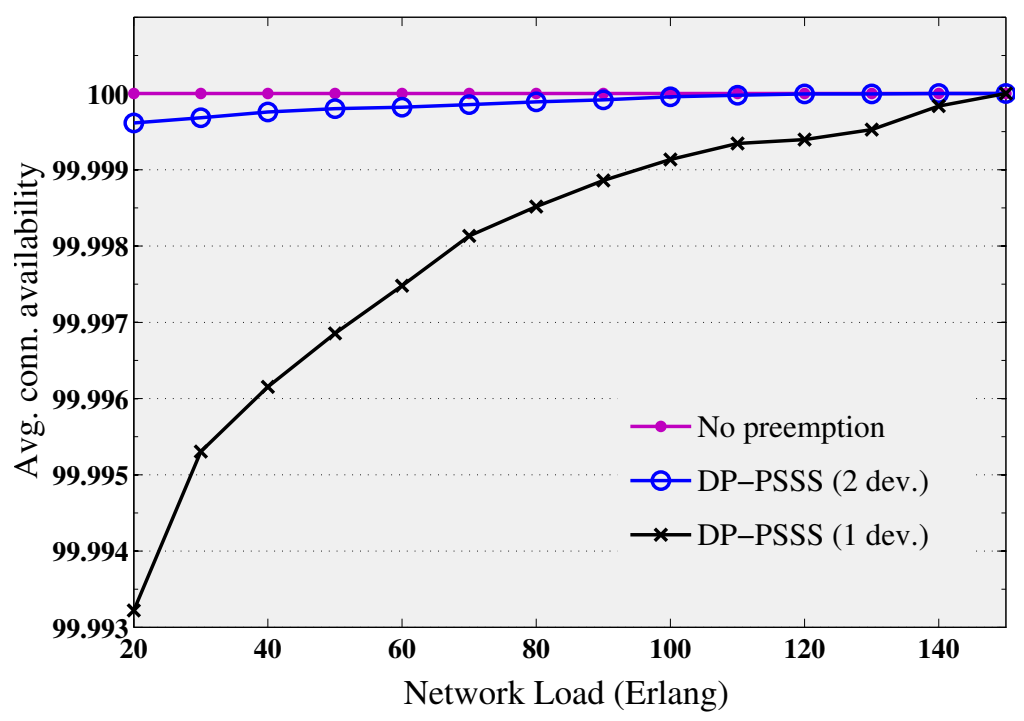

Figure 4.10: Average connection availability vs. offered network load. 


\section{Chapter 5}

\section{Flexgrid Space Division Multiplexing (SDM) Networks}

For decades, the gap between fiber capacity and volume of carried traffic has been so much wider that fiber has been considered as an almost infinitecapacity medium. However, the explosive traffic growth in the last years has brought networks close to the capacity limit of conventional fiber. It is expected that the amount of network traffic will be doubled approximately every 30 months [72], which may require lighting multiple fiber pairs per link in the near future. Although lighting multiple fibers is a feasible solution for addressing the need for additional capacity, it does not deliver the economies of scale with respect to cost and power consumption on which network operators rely. Increasing only the network capacity is not the ultimate goal; the capacity increase must be attained cost-efficiently, such that economies of scale are accomplished.

Given the capacity limits of conventional networks, expansion in fiber capacity can be attained by challenging the underlying system assumptions through innovative techniques. Expanding the transmission band, increasing the number of fiber cores, and/or increasing the number of fiber modes are the approaches that can cost effectively increase fiber capacity. The latter two methods fall under the category of space division multiplexing (SDM), where spatially diverse connections are employed. The SDM can be realized by using multi-mode fiber (MMF), multi-core fiber (MCF), or few-mode multi-core fiber (FM-MCF). MMF employs the propagation of few independent modes within a single core. The number of modes supported by a fiber depends on the core size and the refractive index of the fiber. MMF has a 


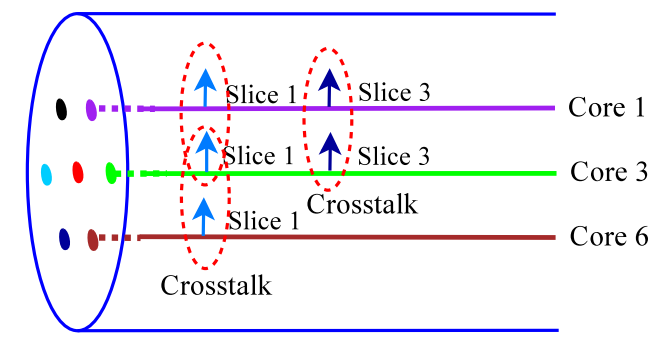

Figure 5.1: Crosstalk between adjacent cores.

larger core size than single-mode fiber (SMF), which allows multiple modes to propagate in the fiber core. On the other hand, MCF has several cores embedded in the fiber cladding where each core acts as a SMF. Ideally, the total fiber capacity increases in proportion to the number of cores, however, this will be more difficult to accomplish as the number of cores increases owing to inter-core crosstalk. To design an MCF with low crosstalk, it is essential to reduce the overlap between the electrical fields of the two cores by increasing the space among them. The number of cores in a round fiber cladding is limited by the cladding diameter due to mechanical reliability considerations [73].

Exploiting the MCF for flexgrid networks necessitates the development of new concepts, such as routing, spectrum and core allocation (RSCA) for connection requests. The introduction of $\mathrm{MCF}$ in the networks mitigates the spectrum continuity constraint of the routing and spectrum assignment (RSA) problem. In fact cores can be switched freely on different links during routing of the connection. Similarly, the route disjointness for demands with same allocated spectrum diminishes to core disjointness at the link level. On the other hand, some new issues such as the inter-core crosstalk should be taken into account while solving the RSCA problem. The intercore crosstalk incurs when optical signals using the same spectrum propagate through adjacent cores in MCF (Figure 5.1). In other words, spectrum overlap among adjacent cores results in limiting the transmission performance of the network. However, the impact of inter-core crosstalk can be alleviated by properly assigning the core and spectrum resources to connection requests.

Paper G investigates the RSCA problem for flexgrid optical networks. The RSCA problem is examined for the network planning case, i.e., all the connection requests to be established in the network are known/given beforehand. The objective is to provision the connections through proper allocation of spectrum and core, while efficiently utilizing the spectrum resources. Paper $\mathbf{H}$ extends the RSCA problem to networks with pro- 


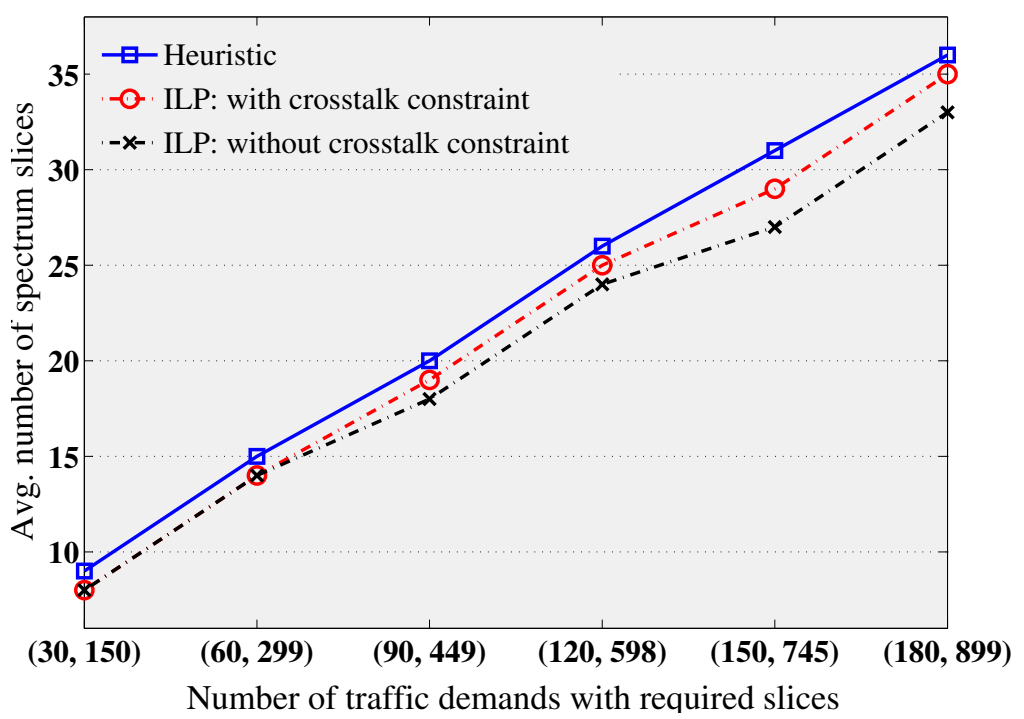

Figure 5.2: Avg. number of spectrum slices per core vs. connection demands.

grammable ROADMS, i.e., AoD nodes. The aim of Paper $\mathbf{H}$ is to compose and synthesize scalable and cost-efficient architectures for SDM networks equipped with AoD nodes by exploring the flexibility inherent in AoD and $\mathrm{MCF}$.

\subsection{Planning Strategy for Flexgrid SDM Networks with MCF}

The proposed planning strategy exploits the flexibility offered by MCF in the form of switching cores on different links during provisioning of connection requests. At the same time, the strategy takes into account the inter-core crosstalk that stems from the multi-cores embedded in the fiber cladding. The design strategy is defined through an ILP formulation and is solved for a real case scenario. To overcome the scalability limitation by the optimal solution based on ILP formulation, a scalable and efficient heuristic algorithm, namely, shortest path with cumulative spectrum availability (SPSA) is proposed. The SPSA sorts the connection requests based on their spectrum requirements and path lengths. The spectrum resources are gradually incremented after each iteration (for establishing connections), till all the requests are provisioned in the network. 


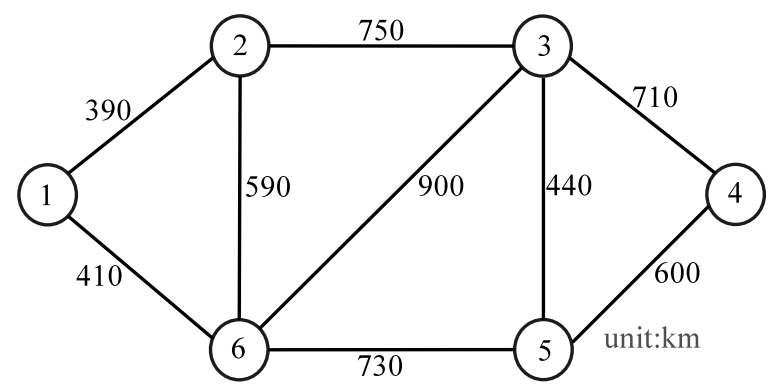

Figure 5.3: Network topology.

Figure 5.2 shows the average number of spectrum slices as a function of the number of connection demands along with total required slices (e.g., 30 connections need a total of 150 slices) to be provisioned in the network for a sample topology (Figure 5.3) with three cores per fiber link. It can be seen from the results that the proposed heuristic is able to well approximate the optimal solution, i.e., average error is less than 6\%. Furthermore, improvement in the number of spectrum slices specially at high loads (for optimal case) can be attained by relaxing the crosstalk constraint. This can be envisaged as a gain brought by the future multi-cores fibers with negligible inter-core crosstalk.

\subsection{Designing Synthetic SDM Network with AoD Nodes}

The optimization problem for a cost-efficient architecture of SDM networks with AoD OXCs is formalized as an ILP problem. The objective is to optimally compose each node and synthesize the whole network with minimum possible resources (MUX/DEMUX) for a given set of connection requests.

Figure 5.4 highlights that significant modules saving is achievable by optimal exploitation of the versatility provided by AoD nodes, and flexibility offered by MCF in the form of switching cores on different links during provisioning of traffic demands. Note that for the benchmark purposes, the number of modules essential to switch signals at each core of the input link to all cores of all the output links (i.e., no fiber switching) is employed. Similarly, Figure 5.5 shows the network power consumption as a function of connection demands. Note that the parameters characterizing the power consumption are set according to the values displayed in the previous chapter (Table 4.1). The figure reveals that by exploiting the flexibility provided by AoD nodes 


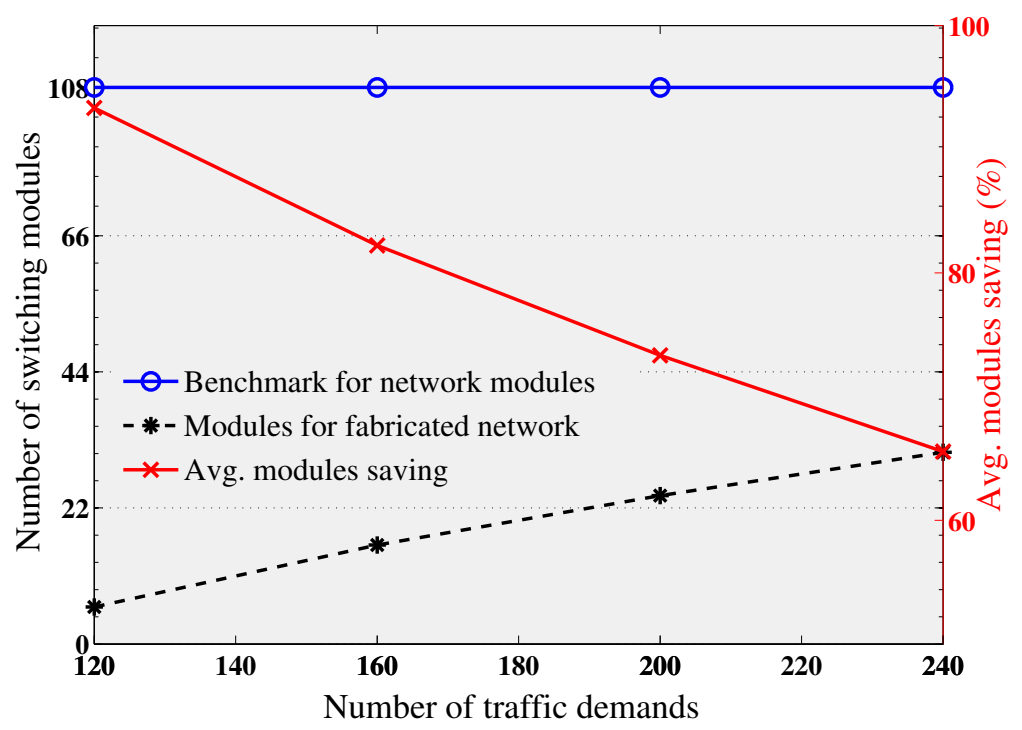

Figure 5.4: Number of switching modules (LHS) and average modules saving (RHS) vs. connection demands;

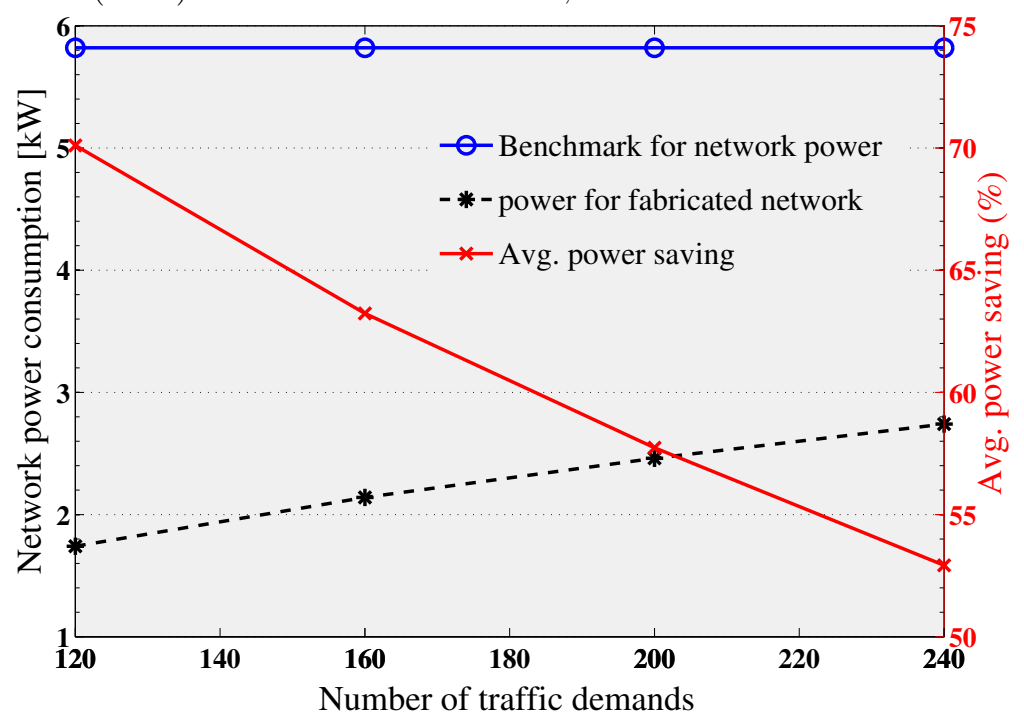

Figure 5.5: Network power consumption (LHS) and average power saving (RHS) vs. traffic demands. 


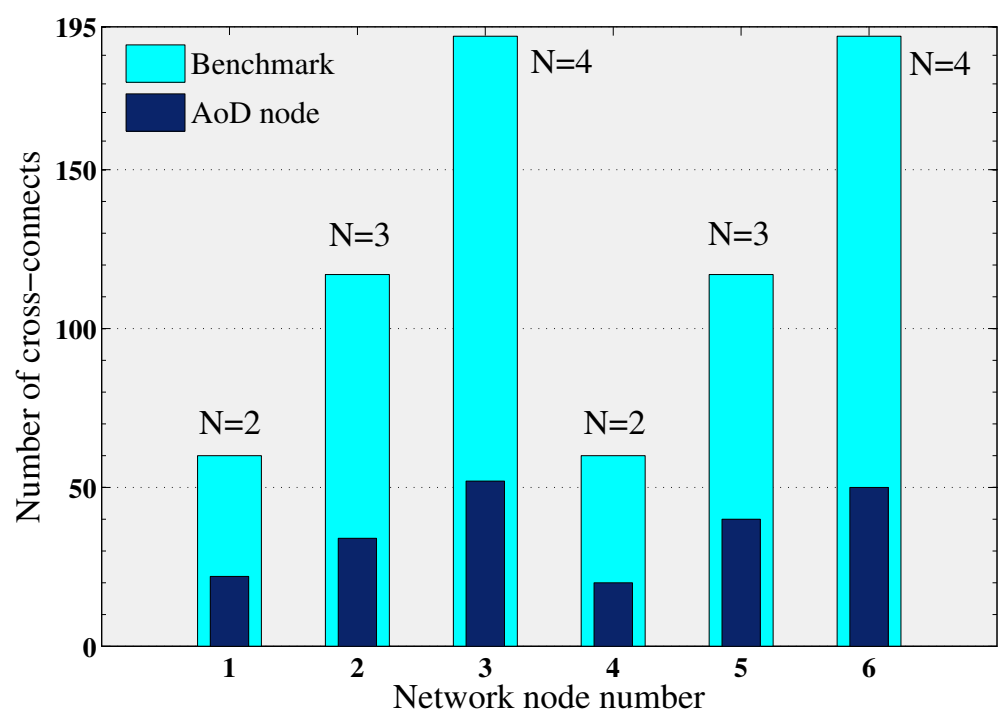

Figure 5.6: Number of cross-connections per node vs. network node number.

the power consumption of the network substantially reduces, compared to the network with rigid design nodes. Finally Figure 5.6 evaluates the number of cross-connections per node required to implement AoD for provisioning a demand of 240 connections. Note that the number of cross-connections indicates the optical backplane port-count, and thus can be considered as a scalability parameter for AoD. The figure confirms that AoD has the capability of reducing the number of cross-connections enormously compared to the static nodes (benchmark). 


\section{Chapter 6}

\section{Summary of Original Work}

\subsection{Contributions of the Thesis}

This thesis generally focuses on circuit-switched core networks with centralized control; however, its contribution is mainly divided into two parts. The first part (Papers A-C) focuses on wavelength-switched dynamic WDM networks. The study analyzes the improvement in network performance when the SLA parameters are fully exploited for lightpaths provisioning, and for scenarios where connection requests belong to one or several service classes. The second part (Papers D-H) investigates the planning and operation of elastic networks with flexible switching. The planning work proposes cost-efficient and energy-efficient design of synthetic networks implemented through AoD. The designed networks are examined for dynamic traffic environment, and several approaches for improving the blocking probability owing to scarcity of switching resources in AoD nodes are presented. The planning issue is further extended to MCF based SDM networks. Design strategies with different objectives, such as minimizing spectrum resources and cost are introduced for SDM networks. These strategies exploit the flexibility offered by MCF in the form of switching cores on different links during provisioning of connections and versatility inherent by $\mathrm{AoD}$ nodes.

Brief summaries of the papers included in this thesis are as follows:

Paper A: Service Differentiated Provisioning in Dynamic WDM Networks Based on set-up Delay Tolerance

Authored by A. Muhammad, C. Cavdar, L. Wosinska, and R. Forchheimer. 
Published in IEEE/OSA Journal of Optical Communications and Networking (JOCN), 2013.

Abstract: Optical networks are expected to provide a unified platform for a diverse set of emerging applications (e.g., three-dimensional TV, digital cinema, e-health, grid computing, etc). The service differentiation will be an essential feature of these networks. Considering the fact that the users have different patience for different network applications denoted by set-up delay tolerance, it will be one of the key parameter for service differentiation. The service differentiation based on set-up delay tolerance will not only enable the network users to select an appropriate service class in compliance with their requirements, but will also provide an opportunity to optimize the network resource provisioning by exploiting this information resulting in an improvement in the overall performance. The improvement in network performance can be further enhanced by exploiting the connection holding-time awareness. However, when multiple classes of services with different set-up delay tolerance are competing for network resources, the connection requests belonging to service classes with higher set-up delay tolerance have better chance to grab the resources and leave less room for the others resulting in degradation in the blocking performance of less patient customers.

This study proposes different scheduling strategies for promoting the requests belonging to smaller set-up delay tolerance service classes, such as giving priority, reserving some fraction of available resources, and augmenting the search space by providing some extra paths. Extensive simulation results show that: 1) priority in the rescheduling queue is not always sufficient for eradicating the degradation effect of high delay tolerant service classes on the provisioning rate of the most stringent service class, 2) by utilizing the proposed strategies resource efficiency and overall network blocking performance improve significantly in all service classes.

Contribution: The contributions consist of the refinement of the initial idea through experiments, implementation of algorithms and drafting of the text.

\section{Paper B: Fair Scheduling of Dynamically Provisioned WDM Con- nections with Differentiated Signal Quality}

Authored by A. Muhammad, C. Cavdar, and P. Monti

Published in the proceedings of The 16th International Conference on Optical Networking Design and Modeling (ONDM), Essex, Colchester, UK, 2012 . 
Abstract: Emerging on-demand applications (e.g., interactive video, ultrahigh definition TV, backup storage and grid computing) are gaining momentum and are becoming increasingly important. Given the high bandwidth required by these applications, Wavelength Division Multiplexing (WDM) networks are seen as the natural choice for their transport technology. Among the various on-line strategies proposed to provision such services, the ones exploiting the connection holding-time knowledge and the flexibility provided by set-up delay tolerance showed a good potential in improving the overall network blocking performance. However, in a scenario where connection requests are grouped in different service classes, the provisioning success rate might be unbalanced towards those connection requests with less stringent requirements, i.e., not all connection requests are treated in a fair way.

This paper addresses the problem of how to guarantee the signal quality and fair provisioning of different service classes, where each class corresponds to a specified target of quality of transmission (QoT). With this objective in mind three fair scheduling algorithms are proposed, each one combining in a different way the concept of both set-up delay tolerance and connection holding-time awareness under dynamic traffic scenario. Proposed solutions are specifically tailored to facilitate the provisioning of the most stringent service class so as to balance the success rate among the different classes. Simulation results confirm that the proposed approaches are able to guarantee a fair treatment reaching up to $99 \%$ in terms of Jain's fairness index, considering per-class success ratio, without compromising the improvements in terms of overall network blocking probability.

Contribution: The contributions are refinement of initial idea through experiments, implementation of algorithms and writing some part of the text.

\section{Paper C: Trading Quality of Transmission for Improved Blocking Performance in All-Optical Networks}

Authored by A. Muhammad, C. Cavdar, L. Wosinska, and R. Forchheimer. Published in the proceeding of The Asia Communications and Photonics Conference (ACP), Beijing, China, 2013.

Abstract: We propose a connection provisioning strategy in dynamic alloptical networks, which exploit the possibility to allow a tolerable signal quality degradation during a small fraction of the holding-time resulting in a significant improvement of blocking performance.

Contribution: The contributions consist of refinement of the initial idea through experiments, implementation of algorithm and writing the text. 


\section{Paper D: An Optimization Model for Dynamic Bulk Provisioning in Elastic Optical Networks}

Authored by A. Muhammad, M. Furdek, P. Monti, L. Wosinska, R. Forchheimer

Published in the proceeding of The Asia Communications and Photonics Conference (ACP), Shanghai, China, 2014.

Abstract: We investigate benefits of setup-delay tolerance in elastic optical networks and propose an optimization model for dynamic and concurrent connection provisioning. Simulation shows that the proposed strategy offers significant improvement of the network blocking performance.

Contribution: The contributions consist of refinement of the initial idea through experiments, implementing the algorithm, performing the experiments and writing the text.

Paper E: Introducing Flexible and Synthetic Optical Networking: Planning and Operation based on Network Function Programmable ROADMs

Authored by A. Muhammad, G. Zervas, N. Amaya, D. Simeonidou, R. Forchheimer

Published in IEEE/OSA Journal of Optical Communications and Networking (JOCN), 2014.

Abstract: Elastic optical networks are envisaged as a promising solution to fulfill the diverse bandwidth requirements for the emerging heterogeneous network applications. To support flexible allocation of spectrum resources the optical network nodes need to be agile. Among the different proposed solutions for elastic nodes, the one based on Architecture of Demand (AoD) exhibits considerable flexibility against the other alternatives. The node modules in case of AoD are not hard-wired, but can be connected/disconnected to any input/output port according to the requirements. Thus, each AoD node and the network (fabricated with AoD nodes) as a whole acts like an optical field-programmable gate array. This flexibility inherent in AoD can be exploited for different purposes, such as for cost-efficient and energy-efficient design of the networks.

This study looks into the cost-efficient network planning issue for synthetic networks implemented through AoD nodes. The problem is formalized as an integer linear programming formulation for presenting the optimal solution. Furthermore, a scalable and effective heuristic algorithm is proposed 
for cost-efficient design and its performance is compared with the optimal solution. The designed networks with AoD nodes are further investigated for a dynamic scenario, and their blocking probability due to limited switching resources in the nodes are examined. To alleviate the blocking performance for the dynamic case, an efficient synthesis strategy along with a scheme for optimal placement of switching resources within the network nodes are presented.

Extensive results show that: 1) even at high loads, the network with AoD nodes achieves saving of switching modules up to $40 \%$ compared to the one with static ROADMs through a proper network design, 2) by diminishing the spectrum selective switches the overall power consumption of the network reduces by more than $25 \%$ for high loads, 3 ) for the dynamic scenario the blocking owing to node modules constraint alleviates significantly by slightly augmenting the switching devices and optimally deploying them within the network nodes.

Contribution: The contributions are discussing the initial idea, implementing the algorithms, performing the experiments and writing the text.

Paper F: Dynamic Provisioning Utilizing Redundant Modules in Elastic Optical Networks Based on Architecture on Demand Nodes

Authored by A. Muhammad, M. Furdek, P. Monti, L. Wosinska, R. Forchheimer

Published in the proceeding of The 40th European Conference on Optical Communications (ECOC), Cannes, France, 2014.

Abstract: Survivable synthetic ROADMs are equipped with redundant switching modules to support failure recovery. The paper proposes a dynamic connection provisioning strategy which exploits these idle redundant modules to provision regular traffic resulting in a substantial improvement in the blocking performance.

Contribution: The contributions consist of refinement of the initial idea through experiments, implementation of algorithm and drafting of the text.

Paper G: Routing, Spectrum and Core Allocation in Flexgrid SDM Networks with Multi-core Fibers

Authored by A. Muhammad, G. Zervas, D. Simeonidou, R. Forchheimer

Published in the proceedings of The 18th International Conference on Optical Networking Design and Modeling (ONDM), Stockholm, Sweden, 2014. 


\begin{abstract}
Space division multiplexing (SDM) over multi-core fiber (MCF) is advocated as a promising technology to overcome the capacity limit of the current single-core optical networks. However, employing the MCF for flexgrid networks necessitates the development of new concepts, such as routing, spectrum and core allocation (RSCA) for traffic demands. The introduction of MCF in the networks mitigates the spectrum continuity constraint of the routing and spectrum assignment (RSA) problem. In fact cores can be switched freely on different links during routing of the network traffic. Similarly, the route disjointness for demands with same allocated spectrum diminishes to core disjointness at the link level. On the other hand, some new issues such as the inter-core crosstalk should be taken into account while solving the RSCA problem.
\end{abstract}

This paper formulates the RSCA network planning problem using the integer linear programming (ILP) formulation. The aim is to optimally minimize the maximum number of spectrum slices required on any core of MCF of a flexgrid SDM network. Furthermore, a scalable and effective heuristic is proposed for the same problem and its performance is compared with the optimal solution. The results show that the proposed algorithm is able to well approximate the optimal solution based on ILP model.

Contribution: The contributions are the initial idea, implementing the algorithms, performing the experiments and writing the text.

\title{
Paper H: Flexible and Synthetic SDM Networks with Multi-core- Fibers Implemented by Programmable ROADMs
}

Authored by A. Muhammad, G. Zervas, G. Saridis, E. H. Salas, D. Simeonidou, R. Forchheimer

Published in the proceeding of The 40th European Conference on Optical Communications (ECOC), Cannes, France, 2014.

\begin{abstract}
This study looks into network planning issues for synthetic MCFbased SDM networks implemented through programmable ROADMs. The results show that significant savings in switching modules and energy can be attained by exploiting the flexibility inherent in programmable ROADM through a proper network design.
\end{abstract}

Contribution: The contributions are refinement of initial idea through experiments, implementation of algorithm and writing the text. 


\subsection{Other Publications}

The author has published the following papers during the study period that are not included in this thesis:

1. A. Muhammad, G. Zervas, N. Amaya, D. Simeonidou, R. Forchheimer, "Cost-Efficient Design of Flexible Optical Networks Implemented by Architecture on Demand", in Proc. of IEEE/OSA OFC, San Francisco, CA, March 2014.

2. A. Muhammad, P. Monti, I. Cerutti, L. Wosinska, P. Castoldi, "Reliability Differentiation in Energy Efficient Optical Networks with Shared Path protection", in Proc. of IEEE Online GreenCom, October 2013.

3. A. Muhammad, R. Forchheimer, "Improving Connections Provisioning in Hybrid Immediate/Advance Reservation WDM Networks", in Proc. of IEEE/OSA ACP, Guangzhou, China, November 2012.

4. A. Muhammad, R. Forchheimer, "Coexistence of Advance and Immediate Reservation in WDM Networks - Some RWA Strategies", in Proc. of IEEE ICTON, Warwick, UK, July 2012.

5. A. Muhammad, R. Forchheimer, L. Wosinska, "Impairment-Aware Dynamic Provisioning in WDM Networks with Set-up Delay Tolerance and Holding-time Awareness", in Proc. of IEEE ICON, Singapore, December 2011.

6. P. Monti, A. Muhammad, I. Cerutti, C. Cavdar, L. Wosinska, P. Castoldi, A. Tzanakaki, "Energy-Efficient Lightpath Provisioning in a Static WDM Network with Dedicated Path Protection", in Proc. of IEEE ICTON, Stockholm, Sweden, June 2011.

7. A. Muhammad, C. Cavdar, L.Wosinska, R. Forchheimer, "Effect of Delay Tolerance in WDM Networks with Differentiated Services", in Proc. of IEEE/OSA OFC, Los Angeles, CA, March 2011.

8. A. Muhammad, P. Monti, I. Cerutti, L. Wosinska, P. Castoldi, A. Tzanakaki, "Energy-Efficient WDM Network Planning with Protection Resources in Sleep Mode" in Proc. of IEEE GLOBECOM, Miami, December 2010 . 


\section{Chapter 7}

\section{Conclusions and Future Work}

\subsection{Conclusions}

This thesis has contributed to service differentiated provisioning in dynamic WDM networks along with the planning and operation related matters for synthetic optical networks implemented by AoD nodes. Furthermore, the design problem for flexible, and synthetic SDM networks with multi-core fibers are also investigated.

For dynamic networks, the time-based service level specifications, such as set-up delay tolerance and connection holding-time have the potential to improve the networks blocking probability if exploited effectively. Similarly, for transparent dynamic networks with optical impairments (specifically the traffic dependent impairments, e.g., crosstalk) connection request is blocked not only due to the resource unavailability but also due to reduced signal quality. Exploring these two metrics for such networks exhibits significant improvement in network blocking. Moreover, for delay impatient applications, another strategy which employs the possibility to tolerate a slight degradation in quality of transmission during a small fraction of the holdingtime, alleviates the blocking probability of such networks.

For scenarios where network connection requests have different set-up delay tolerance or signal quality requirements (i.e., belong to different service classes), the blocking performance of some service classes are not as good as the overall network blocking ratio. The service class that requires relatively low signal quality or high set-up delay tolerance has more chance to be successfully provisioned when compared to the other (more stringent) 
classes. For these scenarios it is important to develop some strategies that improve the network fairness by keeping a balance among the success rate of the different service classes.

Considering the importance of capital and operational expenditures for flexible networks, the second part of the thesis work examines Capex and Opex for synthetic, and evolvable networks with AoD nodes. The flexibility inherent in AoD is utilized for scalable and cost efficient design of networks through proper RSA technique. Significant reduction in switching devices is achievable by a planning strategy that composes and dimensions the network by allocating switching device where and when needed while maximizing fiber switching operation. In addition, such a design strategy also reduces the power consumption of the network. In fact, unlike networks with static ROADMs the power consumption of the AoD based networks depends on traffic demands. Switching modules deployed in AoD nodes if not used by network connection demands can be switched off, thus, reducing power.

The cost-efficient designed networks with AoD are further investigated for the dynamic traffic situation and the degradation of the network blocking performance due to insufficient number of switching modules at AoD nodes is examined. The blocking owing to the scarcity of switching modules in AoD nodes can be reduced by employing an effective synthesis provisioning scheme along with a strategy for optimal location of switching devices in the network nodes. Furthermore, for survivable flexible networks, the modules dedicated for protection can be utilized to serve connection requests that would otherwise be blocked due to the restricted port connectivity. However, the connections established by a redundant module will be preempted in order to protect connections served by a failed working module, which might decrease connection availability. The results depict that such an approach reduces connection blocking by more than $50 \%$ without a drastic impact on the connection availability performance.

The network planning problem is extended to MCF based SDM networks. The proposed strategies for SDM networks aim to provision the connections through proper allocation of spectrum and core while efficiently utilizing the spectrum resources. Moreover, the strategies exploit the flexibility offered by $\mathrm{MCF}$ in the form of switching cores on different links during provisioning of connection requests. At the same time, the strategies take into account the inter-core crosstalk that stems from the multi-cores embedded in the fiber cladding. Finally, the optimal planning strategy for SDM networks is tailored to fit synthetic ROADM based networks with the goal to optimally compose each node and synthesize the whole network with minimum possible 
switching resources (MUX/DEMUX).

\subsection{Future Work}

Several areas are identified for the extension of the thesis work. For instance, it will be useful to develop a scalable heuristic or metaheuristic strategy for dynamic bulk provisioning for elastic optical networks. Similarly, it will be interesting to investigate the dimensioning problem for synthetic ROADM (AoD) based networks considering the incremental traffic model. Moreover, a planning strategy that exploits the additional degree of freedom in the form of modulation level selection on optical reach basis would be beneficial for AoD based flexible networks. For survivable flexible networks with synthetic ROADMs, the issue of optimal allocation of redundant switching resources to nodes of a given network needs to be investigated.

A worthwhile extension for the SDM networks with MCF will be to devise an inter-core crosstalk aware RSCA strategy that takes into account the actual interference among the connections using the same spectrum on adjacent cores. Similarly, the impact of distance-adaptive modulation formats on the performance of SDM networks needs to be analyzed. Furthermore, it will be useful to develop inter-core crosstalk aware scalable and cost-effective planning strategy for synthetic ROADM based SDM networks. Finally, this study of SDM networks should be extended to the dynamic traffic scenario. Dynamic provisioning strategies that exploit the fundamental features of SDM networks and at the same time take into account the additional constraints inflicted by SDM need to be formulated. 


\section{REFERENCES}

[1] R. Essiambre and R. Tkach, "Capacity trends and limits of optical communication networks," Proceedings of the IEEE, vol. 100, no. 5, pp. 1035-1055, May 2012.

[2] T. Wu, "Bandwidth is the new black gold," Time magazine, Mar. 2010.

[3] J. M. Simmons, Optical networks Design and Planning. Springer Publishers, 2014.

[4] A. Chiu, G. Choudhury, and G. Clapp, "Architectures and protocols for capacity efficient, highly dynamic and highly resilient core networks," IEEE/OSA Journal of Optical Commun. and Networking, vol. 4, no. 1, pp. 1-14, Jan. 2012.

[5] B. Ramamurthy, G. N. Rouskas, and K. M. Sivalingam, NextGeneration Internet Architectures and Protocols. Cambridge University Press, 2011.

[6] W. Fawaz, B. Daheb, O. Audouin, M. Du-Pond, and G. Pujolle, "Service level agreement and provisioning in optical networks," IEEE Commun. Magazine, vol. 42, no. 1, pp. 36-43, Jan. 2004.

[7] O. Gerstel, M. Jinno, A. Lord, and S. Yoo, "Elastic optical networking: a new dawn for the optical layer?" IEEE Commun. Magazine, vol. 50, no. 2, pp. 12-20, Feb. 2012.

[8] N. Amaya, G. Zervas, and D. Simeonidou, "Architecture on demand for transparent optical networks," in Proc. 13th International Conference on Transparent Optical Networks (ICTON), Jun. 2011, pp. 1-4.

[9] A. D. Ellis, J. Zhao, and D. Cotter, "Approaching the non-linear shannon limit," Journal of Lightwave Technology, vol. 28, no. 4, pp. 423-433, Feb. 2010. 
[10] P. Winzer, "Spatial multiplexing: The next frontier in network capacity scaling," in Proc. 39th European Conference and Exhibition on Optical Commun. (ECOC), Sep. 2013, pp. 1-4.

[11] J. Sakaguchi, B. J. Puttnam, W. Klaus, Y. Awaji, N. Wada, A. Kanno, T. Kawanishi, K. Imamura, H. Inaba, K. Mukasa, R. Sugizaki, T. Kobayashi, and M. Watanabe, "19-core fiber transmission of 19x100x172-Gb/s SDM-WDM-PDM-QPSK signals at 305 $\mathrm{Tb} / \mathrm{s}$," in Proc. Optical Fiber Commun. Conference and Exposition (OFC/NFOEC), 2012.

[12] H. Takara and A. Sano, "1.01-Pb/s (12 SDM/222 WDM/456 Gb/s) crosstalk-managed transmission with $91.4-\mathrm{b} / \mathrm{s} / \mathrm{Hz}$ aggregate spectral efficiency," in Proc. 38th European Conference and Exhibition on Optical Commun. (ECOC), 2012.

[13] B. Zhu, T. Taunay, M. Fishteyn, X. Liu, S. Chandrasekhar, M. F. Yan, J. M. Fini, E. M. Monberg, and F. V. Dimarcello, "112-tb/s space-division multiplexed DWDM transmission with 14-b/s/hz aggregate spectral efficiency over a 76.8-km seven-core fiber," Optics Express, vol. 19, pp. 16 665-16671, 2011.

[14] H. Takara, H. Ono, Y. Abe, H. Masuda, K. Takenaga, S. Matsuo, H. Kubota, K. Shibahara, T. Kobayashi, and Y. Miaymoto, "1000-km 7-core fiber transmission of $10 \times 96-\mathrm{Gb} / \mathrm{s}$ PDM-16QAM using raman amplification with 6.5 w per fiber," Optics Express, vol. 20, pp. $10100-$ $10105,2012$.

[15] C. Qiao and M. Yoo, "Optical Burst Switching (OBS) - A new paradigm for an optical Internet," Journal of High Speed Networks, vol. 8, pp. 69 84, 1999 .

[16] P. Pavon-Marino and M. Bueno-Delgado, "Add/drop contention-aware rwa with directionless roadms: The offline lightpath restoration case," IEEE/OSA Journal of Optical Commun. and Networking, vol. 4, no. 9, pp. 671-680, Sep. 2012.

[17] R. Ramaswami and K. Sivarajan, "Routing and wavelength assignment in all-optical networks," IEEE/ACM Transactions on Networking, vol. 3, no. 5, pp. 489-500, Oct. 1995.

[18] G. P. Agrawal, Fiber-optic communication systems. John Wiley \& Sons, Inc., 2002. 
[19] C. Saradhi and S. Subramaniam, "Physical layer impairment aware routing (PLIAR) in WDM optical networks: issues and challenges," IEEE Commun. Surveys Tutorials, vol. 11, no. 4, pp. 109-130, quarter 2009.

[20] C. Murthy and M. Guruusamy, WDM optical networks, concepts, design and algorithms. Prentice Hall, 2001.

[21] H. Zang and J. P. Jue, "A review of routing and wavelength assignment approaches for wavelength-routed optical WDM networks," Optical Networks Magazine, vol. 1, pp. 47-60, 2000.

[22] K. M. Chan and T. Yum, "Analysis of least congested path routing in WDM lightwave networks," in Proc. 13th IEEE Networking for Global Commun. (INFOCOM), vol. 2, Jun. 1994, pp. 962-969.

[23] L. Ruan and D. zhu Du, Optical networks - recent advances. Kluwer Acedemic Publishers, 2001.

[24] G. Jeong and E. Ayanoglu, "Comparison of wavelength-interchanging and wavelength-selective cross-connects in multi-wavelength all-optical networks," in Proc. 15th IEEE Annual Joint Conference of the IEEE Computer Societies. Networking the Next Generation, vol. 1, Mar. 1996, pp. 156-163.

[25] E. Karasan and E. Ayanoglu, "Effects of wavelength routing and selection algorithms on wavelength conversion gain in WDM optical networks," IEEE/ACM Transactions on Networking, vol. 6, no. 2, pp. 186196, Apr. 1998.

[26] S. Subramaniam and R. Barry, "Wavelength assignment in fixed routing WDM networks," in Proc. IEEE International Conference on Commun. (ICC), vol. 1, Jun. 1997, pp. 406-410.

[27] X. Zhang and C. Qiao, "Wavelength assignment for dynamic traffic in multi-fiber WDM networks," in Proc. 7th International Conference on Computer Commun. and Networks, Oct. 1998.

[28] S. Azodolmolky, M. Klinkowski, E. Marin, D. Careglio, J. S. Pareta, and I. Tomkos, "A survey on physical layer impairments aware routing and wavelength assignment algorithms in optical networks," Computer Network, vol. 53, no. 7, pp. 926-944, May 2009. 
[29] K. Christodoulopoulos, P. Kokkinos, K. Manousakis, and E. Varvarigos, "Cross layer rwa in wdm networks: Is the added complexity useful or a burden?" in Proc. 11th International Conference on Transparent Optical Networks (ICTON), Jun. 2009, pp. 1-5.

[30] A. Farrel, J.-P. Vasseur, and J. Ash, "A path computation element (PCE)-based architecture," Aug. 2006.

[31] J. Ahmed, C. Cavdar, P. Monti, and L. Wosinska, "A dynamic bulk provisioning framework for concurrent optimization in PCE-Based WDM networks," Journal of Lightwave Technology, vol. 30, no. 14, pp. 2229 2239, Jul. 2012.

[32] E. Rosen and A. Viswanathan, "Multiprotocol label switching architecture," in IETF RFC 3031, Jan. 2001.

[33] A. Szymanski, A. Lason, J. Rzasa, and A. Jajszczyk, "Grade-of-servicebased routing in optical networks," IEEE Commun. Magazine, vol. 45, no. 2, pp. 82-87, Feb. 2007.

[34] C. Cavdar, M. Tornatore, F. Buzluca, and B. Mukherjee, "Shared-path protection with delay tolerance (sdt) in optical wdm mesh networks," Journal of Lightwave Technology, vol. 28, no. 14, pp. 2068 - 2076, jul. 2010.

[35] M. Tornatore, C. Ou, J. Zhang, A. Pattavina, and B. Mukherjee, "PHOTO: an efficient shared-path-protection strategy based on connection-holding-time awareness," Journal of Lightwave Technology, vol. 23, no. 10, pp. 3138-3146, Oct. 2005.

[36] J. Zheng and H. Mouftah, "Routing and wavelength assignment for advance reservation in wavelength-routed WDM optical networks," in Proc. IEEE International Conference on Commun. (ICC), 2002.

[37] N. Charbonneau and V. Vokkarane, "A survey of advance reservation routing and wavelength assignment in wavelength-routed WDM networks," IEEE Commun. Surveys Tutorials, vol. 14, no. 99, pp. 1-28, 2011.

[38] L. Shen, X. Yang, A. Todimala, and B. Ramamurthy, "A two-phase approach for dynamic lightpath scheduling in WDM optical networks," in Proc. IEEE International Conference on Commun. (ICC), Jun. 2007, pp. 2412-2417. 
[39] L. Shen, A. Todimala, B. Ramamurthy, and X. Yang, "Dynamic lightpath scheduling in next-generation WDM optical networks," in Proc. 25th IEEE International Conference on Computer Commun. (INFOCOM), Apr. 2006, pp. 1-5.

[40] A. Iselt, A. Kirstädter, and R. Chahine, "The Role of ASON and GMPLS for the Bandwidth Trading Market," Aug. 2004.

[41] E. Al Sukhni and H. Mouftah, "Distributed holding-time-aware sharedpath-protection provisioning framework for optical networks," in IEEE Symposium on Computers and Commun. (ISCC), Jul. 2009, pp. 730735 .

[42] D. Lucerna, A. Baruffaldi, M. Tornatore, and A. Pattavina, "On the efficiency of dynamic routing of connections with known duration," in Proc. IEEE International Conference on Commun. (ICC), Jun. 2009, pp. 1-6.

[43] Tech. Rep., bit-error rate (BER) for high-speed serial data communication,. [Online]. Available: http://www.analogzone.com/nett1003.pdf

[44] D. Miras, "A survey of network qos needs of advanced internet applications - working document," in Internet2 QoS working group, Nov. 2002 .

[45] Peter J. Winzer and Rene-Jean Essiambre, "High spectral density optical commun. technologies," in Springer, 2010, pp. 103-124.

[46] M. Jinno, H. Takara, and B. Kozicki, "Dynamic optical mesh networks: drivers, challenges, and solutions for the future," in Proc. 35th European Conference and Exhibition on Optical Commun. (ECOC), 2009, pp. 13 .

[47] Y. Wang, X. Cao, and Y. Pan, "A study of the routing and spectrum allocation in spectrum-sliced elastic optical path networks," in Proc. 30th IEEE Networking for Global Commun. (INFOCOM), 2011, pp. 1503-1511.

[48] B. Kozicki, H. Takara, Y. Sone, A. Watanabe, and M. Jinno, "Distanceadaptive spectrum allocation in elastic optical path network (SLICE) with bit per symbol adjustment," in Proc. Optical Fiber Commun. Conference and Exposition (OFC/NFOEC), 2010, pp. 1-3. 
[49] A. Rosa, C. Cavdar, S. Carvalho, J. Costa, and L. Wosinska, "Spectrum allocation policy modeling for elastic optical networks," in Proc. 9th International Conference on High Capacity Optical Networks and Enabling Technologies (HONET), Dec. 2012, pp. 242-246.

[50] Y. Wang and X. Cao, "Multi-granular optical switching: A classified overview for the past and future," IEEE Commun. Surveys Tutorials, vol. 14, no. 3, pp. 698-713, 2012.

[51] P. Ji and Y. Aono, "Colorless and directionless multi-degree reconfigurable optical add/drop multiplexers," in Proc. 19th Annual Wireless and Optical Commun. Conference (WOCC), 2010, pp. 1-5.

[52] A. Devarajan, K. Sandesha, R. Gowrishankar, B. Kishore, G. Prasanna, R. Johnson, and P. Voruganti, "Colorless, directionless and contentionless multi-degree ROADM architecture for mesh optical networks," in Proc. 2nd International Conference on Commun. Systems and Networks (COMSNETS), 2010, pp. 1-10.

[53] S. Gringeri, B. Basch, V. Shukla, R. Egorov, and T. Xia, "Flexible architectures for optical transport nodes and networks," IEEE Commun. Magazine, vol. 48, no. 7, pp. 40-50, 2010.

[54] N. Amaya, G. Zervas, and D. Simeonidou, "Introducing node architecture flexibility for elastic optical networks," IEEE/OSA Journal of Optical Commun. and Networking, vol. 5, no. 6, pp. 593-608, Jun. 2013.

[55] M. Jinno, H. Takara, Y. Sone, K. Yonenaga, and A. Hirano, "Multiflow optical transponder for efficient multilayer optical networking," IEEE Commun. Magazine, vol. 50, no. 5, pp. 56-65, 2012.

[56] D. Geisler, N. Fontaine, R. Scott, L. Paraschis, O. Gerstel, and S. Yoo, "Flexible bandwidth arbitrary modulation format, coherent optical transmission system scalable to terahertz BW," in Proc. 37th European Conference and Exhibition on Optical Commun. (ECOC), Sep. 2011, pp. 1-3.

[57] S. K. Korotky, R.-J. Essiambre, and R. W. Tkach, "Expectations of optical network traffic gain afforded by bit rate adaptive transmission," Bell Labs Technical Journal, vol. 14, no. 4, pp. 285-295, 2010.

[58] V. Lopez, O. Gonzalez de Dios, O. Gerstel, N. Amaya, G. Zervas, D. Simeonidou, and J. Fernandez-Palacios, "Target cost for sliceable 
bandwidth variable transponders in a real core network," in Future Network and Mobile Summit (FutureNetworkSummit), Jul. 2013, pp. $1-9$.

[59] M. Casado, M. J. Freedman, J. Pettit, J. Luo, N. McKeown, and S. Shenker, "Ethane: Taking control of the enterprise," SIGCOMM Computer Commun. Review, vol. 37, no. 4, pp. 1-12, Aug. 2007.

[60] N. Sambo, F. Cugini, G. Bottari, P. Iovanna, and P. Castoldi, "Distributed setup in optical networks with flexible grid," in Proc. 37th European Conference and Exhibition on Optical Commun. (ECOC), Sep. 2011, pp. 1-3.

[61] "Spectral grids for WDM applications: DWDM frequency grid," in ITUT Recommendation G.694.1 (ed. 2.0), Feb. 2012.

[62] G. Meloni, F. Paolucci, N. Sambo, F. Cugini, M. Secondini, L. Gerardi, L. Poti, and P. Castoldi, "PCE architecture for flexible WSON enabling dynamic rerouting with modulation format adaptation," in Proc. 37th European Conference and Exhibition on Optical Commun. (ECOC), Sep. 2011, pp. 1-3.

[63] Y. Sone, A. Hirano, A. Kadohata, M. Jinno, and O. Ishida, "Routing and spectrum assignment algorithm maximizes spectrum utilization in optical networks," in Proc. 37th European Conference and Exhibition on Optical Commun. (ECOC), Sep. 2011, pp. 1-3.

[64] Y. Yin, M. Zhang, Z. Zhu, and S. Yoo, "Fragmentation-aware routing, modulation and spectrum assignment algorithms in elastic optical networks," in Proc. Optical Fiber Commun. Conference and Exposition (OFC/NFOEC), Mar. 2013, pp. 1-3.

[65] A. Castro, L. Velasco, M. Ruiz, J. Pedro, and D. Careglio, "Dynamic routing and spectrum (re)allocation in future flexgrid optical networks," Computer Networks., vol. 56, no. 12, pp. 2869-2883, Aug. 2012.

[66] F. Cugini, F. Paolucci, G. Meloni, G. Berrettini, M. Secondini, F. Fresi, N. Sambo, L. Poti, and P. Castoldi, "Push-pull defragmentation without traffic disruption in flexible grid optical networks," Journal of Lightwave Technology, vol. 31, no. 1, pp. 125-133, Jan. 2013.

[67] M. Zhang, Y. Yin, R. Proietti, Z. Zhu, and S. Yoo, "Spectrum defragmentation algorithms for elastic optical networks using hitless spectrum 
retuning techniques," in Proc. Optical Fiber Commun. Conference and Exposition (OFC/NFOEC), Mar. 2013, pp. 1-3.

[68] X. Yu, J. Zhang, Y. Zhao, T. Peng, Y. Bai, D. Wang, and X. Lin, "Spectrum compactness based defragmentation in flexible bandwidth optical networks," in Proc. Optical Fiber Commun. Conference and Exposition (OFC/NFOEC), Mar. 2012, pp. 1-3.

[69] X. Wang, Q. Zhang, I. Kim, P. Palacharla, and M. Sekiya, "Utilization entropy for assessing resource fragmentation in optical networks," in Proc. Optical Fiber Commun. Conference and Exposition (OFC/NFOEC), 2012, pp. 1-3.

[70] Finisar's Waveshaper. [Online]. Available: http://www.finisar.com

[71] Calient's Fiber Connect. [Online]. Available: http://www.calient.net/products/

[72] Tech. Rep., Cisco Visual Networking Index: Forecast and Methodology, 2010-2015.

[73] K. Nakajima, P. Sillard, D. Richardson, M.-J. Li, R.-J. Essiambre, and S. Matsuo, "Transmission media for an SDM-based optical communication system," IEEE Comm. Magazine, vol. 53, no. 2, pp. 44-51, Feb. 2015. 


\section{Part II}

\section{Wavelength Switched Optical \\ Networks}





\section{Papers}

The articles associated with this thesis have been removed for copyright reasons. For more details about these see:

http://urn.kb.se/resolve?urn=urn:nbn:se:liu:diva-115908 


\section{Part III}

\section{Elastic Optical Networks}





\section{Papers}

The articles associated with this thesis have been removed for copyright reasons. For more details about these see:

http://urn.kb.se/resolve?urn=urn:nbn:se:liu:diva-115908 